\title{
Small RNA sequencing reveals a role for sugarcane miRNAs and their targets in response to Sporisorium scitamineum infection
}

Yachun Su, Yuye Zhang, Ning Huang, Feng Liu, Weihua Su, Liping Xu, Waqar Ahmad, Qibin Wu, Jinlong Guo and Youxiong Que*

\begin{abstract}
Background: Sugarcane smut caused by Sporisorium scitamineum leads to a significant reduction in cane yield and sucrose content. MicroRNAs (miRNAs) play an important role in regulating plant responses to biotic stress. The present study was the first to use two sugarcane genotypes, YA05-179 (smut-resistant) and ROC22 (smut-susceptible), to identify differentially expressed miRNAs in sugarcane challenged with S. scitamineum by using high-throughput sequencing.

Results: The predicted target gene number corresponding to known differentially expressed miRNAs in YA05-179 was less than that in ROC22, however most of them were in common. Expression of differential miRNAs under S. scitamineum challenge was mostly downregulated, with similar trends in the two varieties. Gene ontology (GO) analysis showed that the target gene classification of known miRNAs was similar to that of the newly identified miRNAs. These were mainly associated with cellular processes and metabolic processes in the biological process category, as well as combination and catalytic activity in the molecular function category. Kyoto Encyclopedia of Genes and Genomes (KEGG) pathway enrichment analysis revealed that these predicted target genes involved in a series of physiological and biochemical pathways or disease resistance-related physiological metabolism and signal transduction pathways, suggesting that the molecular interaction mechanism between sugarcane and S. scitamineum was a complex network system. These findings also showed certain predicted target genes of miR5671, miR5054, miR5783, miR5221, and miR6478 play roles in the mitogen-activated protein kinase (MAPK) signaling pathway, plant hormone signal transduction, and plant-pathogen interaction. Quantitative real-time PCR (qRT-PCR) analysis showed that majority of the known miRNAs and its predicted target genes followed a negatively regulated mode. Seven out of eight predicted target genes showed identical expression after $12 \mathrm{~h}$ treatment and reached the highest degree of matching at $48 \mathrm{~h}$, indicating that the regulatory role of miRNAs on the target genes in sugarcane was maximized at $48 \mathrm{~h}$ after $\mathrm{S}$. scitamineum challenge.
\end{abstract}

Conclusions: Taken together, our findings serve as evidence for the association of miRNA expression with the molecular mechanism underlying the pathogenesis of sugarcane smut, particularly on the significance of miRNA levels in relation to the cultivation of smut-resistant sugarcane varieties.

Keywords: Saccharum spp., Sporisorium scitamineum, High-throughput sequencing, miRNA, Function prediction of target genes, qRT-PCR

\footnotetext{
* Correspondence: queyouxiong@126.com

Key Laboratory of Sugarcane Biology and Genetic Breeding, Ministry of

Agriculture, Fujian Agriculture and Forestry University, Fuzhou 350002, China
}

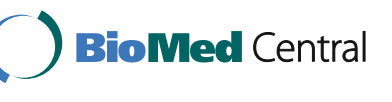

(c) The Author(s). 2017 Open Access This article is distributed under the terms of the Creative Commons Attribution 4.0 International License (http://creativecommons.org/licenses/by/4.0/), which permits unrestricted use, distribution, and reproduction in any medium, provided you give appropriate credit to the original author(s) and the source, provide a link to the Creative Commons license, and indicate if changes were made. The Creative Commons Public Domain Dedication waiver (http://creativecommons.org/publicdomain/zero/1.0/) applies to the data made available in this article, unless otherwise stated. 


\section{Background}

Sugarcane is the most important sugar crop in China, accounting for $92 \%$ of the total sugar production in the country. To date, sugarcane smut caused by Sporisorium scitamineum widely occurs in sugarcane fields worldwide and has become one of the most difficult fungal diseases to control [1, 2]. Due to poor resistance to sugarcane smut, several prevalent sugarcane varieties in China such as NCO310, F134, and CP73-351 have become vulnerable to smut disease, thereby resulting in major losses in cane yield and sugar and thereby have been eliminated in succession [2]. The cultivation of smut-resistant sugarcane varieties is considered as the most cost-effective approach in controlling this disease [3]. Hence, in-depth studies of disease resistance mechanisms are imperative for the prevention and control of sugarcane smut.

Extensive studies on the mechanism of interaction between sugarcane and S. scitamineum have been conducted in the past few years [4-16]. Heinzeetal et al. [4] used suppression subtractive hybridization ( $\mathrm{SSH}$ ) to obtain two full-length genes that were potentially important for the interaction between sugarcane and S. scitamineum, which in turn could facilitate in the evaluation of the two molecular markers for sugarcane smut resistant varieties. Borrás et al. [5] used cDNA-amplified fragment length polymorphism (cDNA-AFLP) technology to study the differential gene expression of sugarcane after the development of smut disease and screened 62 differentially expressed genes, including 52 upregulated genes and ten downregulated genes. Among these 52 upregulated genes, 19 were directly related to biological functions such as defense and signal transmission. Que et al. [6] used twodimensional gel electrophoresis (2DE), matrix-assisted laser desorption/ionization time-of-flight mass spectrometry (MALDI-TOF MS), and quantitative PCR to comprehensively analyze the molecular responses, including transcription and protein expression in relation to the interaction between sugarcane and S. scitamineum. By RNA sequencing and isobaric tags for relative and absolute quantitation (iTRAQ), the gene and protein expression profiles of sugarcane in response to the infection of S. scitamineum were constructed, and its relationship with the mechanism of interaction between sugarcane and S. scitamineum was identified [7]. In 2015, Su et al. [8] developed a rapid and visual loop-mediated isothermal amplification (LAMP) for the detection of $S$. scitamineum in sugarcane. This assay was nearly 100 times more sensitive than conventional PCR for detection of this pathogen [8]. Su et al. also obtained three members of the $\beta$-1,3-glucanase gene family (ScGluA1, ScGluD1 and ScGluD2) [9, 10], one catalase gene (ScCAT1) [11] and ten chitinase family genes [12], which are associated with the pathogenicity of sugarcane smut. Wu et al. [13] used Solexa high-throughput sequencing for differential gene expression profiling of sugarcane after infection with sugarcane smut and 2,015 differentially expressed sequence tags (ESTs) were screened, including three upregulated ESTs that were related to the mitogenactivated protein kinase (MAPK) signaling pathways. In addition, our group was the first to report the wholegenome sequence of $S$. scitamineum and to comprehensively describe the pathogenesis of sugarcane smut [14], and two other groups, Taniguti et al. [15] and Dutheil et al. [16], followed suit. It is the fourth smut fungal species subjected to whole-genome sequencing, following Ustilago maydis and S. reilianum in maize $[17,18]$ and Ustilago hor$d e i$ in barley [19]. The aforementioned findings have promoted research on the molecular response involved in the interaction between sugarcane and smut fungus. However, no mechanistic analysis of microRNA (miRNA) differential expression and functional analysis of the potential target gene of sugarcane under S. scitamineum challenge has been conducted to date.

miRNAs are a class of non-coding small RNA (sRNA) of unequal lengths that ranging from 20 to 25 nt [20-22]. These have important biological functions, and its mediated post-transcriptional gene regulation is an extremely important sRNA regulatory pathway in in vivo models [20-22]. In 1993, Lee et al. [23] first described a miRNA in Caenorhabditis elegans by showing that an sRNA fragment that was complementary to the lin-14 genomic sequence was a lin-14 transcript, which could negatively regulate lin-14 expression. In 2000, Reinhar et al. [24] found a gene, let-7, in C. elegans with a similar function as that of lin-14, further confirming that miRNA-mediated transcriptional regulation commonly but not coincidentally existed in organisms. Ruvkun et al. [25] in 2004 described a novel posttranscriptional gene expression regulatory mechanism that involved miRNA target genes. Since then, a large number of novel miRNAs have been reported in various plant and animal species, including human [26], mouse [27], Drosophila [28], nematodes, and Arabidopsis [29].

Previous studies have shown that miRNAs do not only influence the growth and development of organisms by regulating transcription factors, but also degrade target gene mRNAs or stop the target gene translation to change the cellular behavior of plants through numerous physiological pathways such as protein hydrolysis, metabolism, and ion transport [30], as well as signal transduction pathways [31-33]. Compared to miRNAs in animals, studies on plant miRNAs have lagged behind and only begun in 2002 [34]. Napoli et al. [35] confirmed the presence of miRNAs and small interfering RNAs (siRNAs), including its mechanism of interaction. They also explained the specific mechanism underlying co-suppression during the synthesis of flavonols and anthocyanins in plants [35]. A subsequent study showed that miRNAs played important biological functions in plants, wherein they are not only involved in various physiological and biochemical 
processes and regulate normal growth and development of organisms by controlling the expression of transcription factors [30-33], but also had a regulatory role in abiotic or biotic stresses [36, 37]. Jones et al. [38] showed that plants subjected to drought, cold, and high salinity stress induced changes in the expression of miR319c, miR393, miR395, miR397b, and miR402. For example, the ATP sulfurylase 1 gene (APS1), a target gene of miR395, significantly decreased under low sulfate stress. Patade et al. [39] revealed that sugarcane miR159 plays an important role in its response to high salinity stress. Lu et al. [40] obtained 48 miRNA sequences from poplars whose target genes were associated with growth and development, stress responses, anti-virus infection, and also other life-related processes. A previous study showed correlations between miRNA and virus-induced illness, as well as virus-mediated gene silencing [41]. Kasschau et al. [42] demonstrated that overexpression of the helper component-proteinase (Hc-Pro) gene in Arabidopsis plants significantly reduced miR171 expression and further increased target gene expression of miR171, thereby resulting in miR171-related developmental deficiency in plants.

This study focused on the interaction of two different sugarcane genotypes YA05-179 (smut resistant) and ROC22 (smut susceptible) inoculated with S. scitamineum $48 \mathrm{~h}$ by conducting differential miRNA expression analysis and quantitative real-time PCR (qRT-PCR) validation to identify and analyze the expression patterns of miRNA in sugarcane during S. scitamineum challenge, as well as functional analysis to predict its target genes. Moreover, the present study provides evidence on the role of miRNA against $S$. scitamineum challenge in sugarcane to further broaden and strengthen our understanding of the molecular mechanism underlying the response of sugarcane against smut disease, thereby providing a theoretical basis for the cultivation of smutresistant sugarcane varieties.

\section{Methods}

Plant growth and stress treatment

Smut spores were collected from the host variety of ROC22, which were propagated at the Key Laboratory of Sugarcane Biology and Genetic Breeding, the Ministry of Agriculture/Fujian Agriculture and Forestry University (Fuzhou, China). The smut spores were placed in a paper bag, air-dried, and subsequently stored in a sealed container at $4{ }^{\circ} \mathrm{C}$ until use. The sugarcane varieties used in the experiments, ROC22 (smut-susceptible genotype) and YA05-179 (smut-resistant genotype), were provided by the Key Laboratory of Sugarcane Biology and Genetic Breeding, Ministry of Agriculture/Fujian Agriculture and Forestry University. Healthy and uniform sugarcane (from the $4^{\text {th }}$ to $7^{\text {th }}$ nodes counting from the basal node) were collected and cut into single-bud setts, followed by soaking in clean running water for $1 \mathrm{~d}$. The treated materials were then incubated at $28{ }^{\circ} \mathrm{C}$ and cultivated in a moisturizer until the sugarcane buds grew to $1-2-\mathrm{cm}$ in length, followed by needle puncture inoculation of smut spores suspension $\left(5 \times 10^{6}\right.$ spores $/ \mathrm{mL}$, with $0.01 \%$ volume ratio of Tween-20) into the sugarcane buds. The control groups were injected with sterile water (with $0.01 \%$ volume ratio of Tween-20). After treatment, the sugarcane buds of the two groups were continuously incubated at $28{ }^{\circ} \mathrm{C}$ with conditions of $12 \mathrm{~h}$ light and $12 \mathrm{~h}$ dark photoperiods [8]. To minimize biological variance, three sugarcane buds were collected at each time point (i.e., 0,12 , 48, and $96 \mathrm{~h}$ ) after inoculation and mixed well, followed by snap freezing in liquid nitrogen and storing at $-80^{\circ} \mathrm{C}$ until use.

\section{RNA isolation and sequencing}

Total RNA was extracted from the ROC22 and YA05-179 sugarcanes in the treated and control groups using TRIzol $^{\mathrm{TM}}$ (Invitrogen, Carlsbad, CA, USA). RNA integrity was determined by $1 \%$ agarose gel electrophoresis. The concentration of total RNA was determined by using an Agilent Bio-analyzer 2100 system (Agilent Technologies, Santa Clara, CA, USA). The samples of ROC22 and YA05179 inoculated with sterile water and smut spores for $48 \mathrm{~h}$, namely, RCK (control group of ROC 22), RT (treatment group of ROC22), YACK (control group of YA05-179), and YAT (treatment group of YA05-179) were used for the construction of sRNA libraries (Beijing Genomics Institute, Beijing, China). sRNA fragments (18-30 nt) collected from 15\% PAGE were each connected to $5^{\prime}$ end and $3^{\prime}$ end adapters, followed by reverse transcription and PCR analysis and were then sequenced using a HiSeq2000 sequencing system.

\section{Sequencing data processing and analysis}

The adapters at both ends of the 50-nt fragments obtained from the high-throughput sequencing were detached, followed by removal of contaminating sequences and lowquality reads to obtain clean reads. Then, statistical analysis of sequence length distribution, as well as common and specific sequences among samples was performed. The length of sRNA ranged from 18 to $30 \mathrm{nt}$, with miRNAs normally 21-22 nt in length, siRNA $21-24 \mathrm{nt}$, and Piwiinteracting RNA (piRNA) within the range of $28-30 \mathrm{nt}$ [43]. The peak value representing the quantity of different lengths of sRNAs was either $21 \mathrm{nt}$ or $24 \mathrm{nt}$ in length for plant samples, whereas the peak value of sRNAs of animal samples was $22 \mathrm{nt}$ [43]. Classification and annotation of clean sequences were performed to generate information on each component and expression levels of each sample. Using GenBank (http://www.ncbi.nlm.nih.gov/) and Rfam 10.1 (http://rfam.sanger.ac.uk) databases comparison, noncoding RNAs such as ribosomal RNA (rRNA), transfer RNA (tRNA), small nuclear RNA (snRNA), snoRNA, 
and signal recognition particle RNA (srpRNA) were identified and removed. The remaining sRNAs were subjected to a Basic Local Alignment Search Tool (BLASTn) search with no more than two mismatches against miRBase 18.0 database (http://www.mirbase.org/) to identify known miRNA in the samples [44, 45]. sRNA annotation followed a priority rule for classification to avoid redundancy: non-coding RNA (in which GenBank $>$ Rfam) $>$ known miRNA > repeat [46]. Due to the lack of genome information for sugarcane, the sequences that did not match known miRNAs were mapped to the Sugarcane_Unigene database $(65,852$ unigenes) established by our previous transcriptome analysis in ROC22 and YA05-179 post-S. scitamineum infection for 24, 48 and $120 \mathrm{~h}$ [47] and the sugarcane EST in GenBank and the $S$. scitamineum genome database [14] to identify potentially novel miRNA candidates. Mireap (http://sourceforge.net/projects/mireap/) with default parameters was used to predict the sRNAs without annotation and prepare the secondary structure of the novel miRNA.

Differential expression of S. scitamineum-responsive miRNAs To understand the differential expression of sugarcane miRNA after S. scitamineum challenge, the observed frequencies of unique sequences were normalized to the reads per million (RPM) data. If the original miRNA expression in a library was zero, the normalized read count of this miRNA was adjusted to 0.01 in the library for further calculation [48-50]. We performed statistical analysis of known miRNAs, as well as novel miRNAs to identify significant differences in the expression between the treatment and control groups (comparisons: RT vs. RCK or YAT vs. YACK). Then we used the graphs of $\log _{2}$-ratio and scatterplot to compare the expression level of miRNAs expressed by both groups. The specific procedures are as follows: (1) treatment and control groups were normalized to the same orders of magnitude. Formula: Normalized expression level $=$ miRNA expression level/total expression level of the sample $\times$ normalized magnitude; (2) Normalized results were used to calculate the fold change and $P$-value, as well as for graph preparation. The formula for calculating fold change was as follows: Fold change $=\log _{2}$ (treatment vs. control). The $P$-value was calculated based on the following equation [51]:

$$
p(y \mid x)=\left(\frac{N 2}{N 1}\right)^{y} \frac{(x+y) !}{x ! y !\left(1+\frac{N 2}{N 1}\right)^{(x+y+1)}} \quad \begin{aligned}
& C\left(y \leq y_{\text {min }} \mid x\right)=\sum_{y=0}^{y \leq y_{\min }} p(y \mid x) \\
& D\left(y \geq y_{\text {max }} \mid x\right)=\sum_{y \geq y_{\max }}^{\infty} p(y \mid x)
\end{aligned}
$$

where $x$ means control, $y$ means treatment, $N_{1}$ means the normalized expression of a miRNA in the control library, and $N_{2}$ means the normalized expression of the same miRNA in the treatment library. C and D are used to estimate the confidence intervals $\left[y_{\min }, y_{\max }\right]$ in regards to a specific $P$-value.

The value of fold change $>1$ or $<-1$ and $P$-value $<0.05$ were used as criteria in screening for miRNAs that were significantly differentially expressed between samples. In addition, the present study performed cluster analysis of differentially expressed miRNAs in the two sugarcane varieties, i.e., cluster analysis was performed to identify differentially expressed known and novel miRNAs in the treated sugarcane samples after S. scitamineum challenge and the control samples inoculated with sterile water [52].

\section{miRNA target gene prediction and functional analysis}

With reference to the Sugarcane_Unigene database (65,852 unigenes) [47] and the sugarcane EST in GenBank, the present study used the psRNATarget online software (http://plantgrn.noble.org/psRNATarget/) to predict the target genes of the known miRNAs and the novel miRNAs. The specific prediction standards were based on Allen et al. [53] and Schwab et al. [54]. Then, these predicted target genes of differentially expressed miRNAs in RT/RCK and YAT/YACK were subjected to Gene Ontology (GO, http://www.geneontology.org/) enrichment and Kyoto Encyclopedia of Genes and Genomes (KEGG, http://www.genome.jp/kegg/) pathway analyses [55].

\section{Validation of miRNAs and its predicted target genes by qRT-PCR analysis}

qRT-PCR was performed to determine the expression levels of 20 differentially expressed miRNAs. These 20 miRNAs included 16 known miRNAs and 4 novel miRNAs. Primer 5.0 software was used to design upstream primers (Additional file 1: Table S1), whereas downstream primers were derived from the Uni-miR qPCR Kit purchased from Takara (Dalian, China). The internal reference was $5 S$ rRNA [56]. The sugarcane buds from ROC22 and YA05-179 inoculated with distilled water and S. scitamineum at 0 and $48 \mathrm{~h}$ were used for qRTPCR samples. Reverse transcription was conducted using the One Step PrimeScript ${ }^{\bullet}$ miRNA cDNA Synthesis Kit (Perfect Real Time) (Takara, China), following the manufacturer's instructions. Polyadenylation reaction was used to detect miRNA expression. The $2^{-\Delta \Delta C t}$ method [57] was used to calculate the miRNA expression levels of ROC22 and YA05-179 at $48 \mathrm{~h}$ after S. scitamineum infection. Moreover, qRT-PCR was used to determine the expression patterns of the 12 miRNAs in ROC22 and YA05-179 at 0, 12, 48, and $96 \mathrm{~h}$ after S. scitamineum challenge. 
Beacon Designer 7.0 software was used to design the quantification primers of the 23 randomly selected miRNA target genes (Additional file 2: Table S2). qRTPCR was used to analyze the expression patterns of the predicted target genes in ROC22 and YA05-179 at 0, 12, 48, and $96 \mathrm{~h}$ after $S$. scitamineum challenge. The glyceraldehyde-3-phosphate dehydrogenase (GAPDH) gene was used as internal reference [58]. Reverse transcription was performed using the PrimeScript ${ }^{\mathrm{TM}} \mathrm{RT}$ PCR Kit (Takara, Dalian, China), following the manufacturer's instructions. For qRT-PCR expression analysis, the total volume of reaction system was $20 \mu \mathrm{L}$, which included $10 \mu \mathrm{L}$ SYBR Premix Ex Taq $^{\mathrm{TM}}$ II $(2 \times)$ (Takara), $0.8 \mu \mathrm{L}$ each of the upstream and downstream primers $(10 \mu \mathrm{M})$, $1 \mu \mathrm{L}$ of the cDNA template, and $7.4 \mu \mathrm{L}$ of $\mathrm{ddH}_{2} \mathrm{O}$. The reaction conditions were $50{ }^{\circ} \mathrm{C}$ for $2 \mathrm{~min}$ and $95^{\circ} \mathrm{C}$ for $60 \mathrm{~s}$; followed by 40 cycles of $95{ }^{\circ} \mathrm{C}$ for $15 \mathrm{~s}$ and $60{ }^{\circ} \mathrm{C}$ for $60 \mathrm{~s}$; and $95{ }^{\circ} \mathrm{C}$ for $15 \mathrm{~s} ; 60{ }^{\circ} \mathrm{C}$ for $60 \mathrm{~s} ; 95^{\circ} \mathrm{C}$ for $15 \mathrm{~s}$. All assays were performed in triplicate. At the end of amplification, the $2^{-\Delta \Delta C t}$ method [57] was used to calculate the results of qRT-PCR analysis. Statistical analysis was conducted using the Data Processing System (DPS) v7.05 software (China). Data were expressed as the mean \pm standard error $(\mathrm{SE})$. Significance $(P$-value $<0.05)$ was calculated using one-way Analysis of Variance (ANOVA) followed by Duncan's new multiple range test.

\section{Results}

\section{Categories and size distribution of sRNAs in sugarcane after challenging with $S$. scitamineum}

Sequence impurities generated from sRNA sequencing generally refers to contaminated sequences such as noninsert fragments, fragments without $3^{\prime}$ adapters or fragments with only $5^{\prime}$ adapters, reads containing polyA sequences, and sequences with lengths $<18$ nt [59]. In the present study, the four samples, RCK, RT, YACK, and YAT, underwent sequencing and all obtained $3 \times 10^{8}$ high-quality $18-30$ nt clean reads, in which $36,396,588$, 27,812,972, 27,464,468, and 28,290,231 reads were from each library, respectively (Additional file 3: Table S3). Additional file 4: Figure S1 shows that the tested sRNA sequence length of the four samples were mainly within the range of 20-24 nt, a typical size of Dicer-derived sRNAs, in which, majority of the reads were $24 \mathrm{nt}$ in length, and accounted for 45.09, 54.26, 55.80, and $59.24 \%$ of the read in RACK, RT, YACK, and YAT, respectively. The proportions of 20-22 nt sRNAs in the treated sugarcane samples of ROC22 and YA05-179 after S. scitamineum challenge were relatively less than that observed in the controls, whereas the proportion of 23 nt-24 nt sRNAs in the treated sugarcane samples of ROC22 and YA05-179 after S. scitamineum challenge were relatively higher than that of the controls. These findings indicated that S. scitamineum induces changes in the expression pattern of sRNAs in both sugarcane genotypes.

Comparison of the sRNA libraries of the S. scitamineum-treated and the control groups (RT vs. RCK and YAT vs. YACK) indicated that only less than $15 \%$ of the unique reads were shared by the two sRNA libraries. Figure $1 \mathrm{a}$ and $\mathrm{b}$ show that the common sequences between RT and RCK accounted for $64.47 \%$ of the total reads and $13.27 \%$ of the unique reads, respectively. Figure $1 \mathrm{c}$ and $\mathrm{d}$ show that the common sequences between YAT and YACK accounted for $58.23 \%$ of the total reads and $12.88 \%$ of the unique reads. The observation of unique reads with broad specificity suggests that these may be related to the resistant phenotype of sugarcane to S. scitamineum.

The sRNAs obtained in the present study were concentrated within the sense and antisense strands of rRNA and sense strand of tRNA (Additional file 5: Table S4). Matching of the sRNA sequences of small cytoplasmic RNA (scRNA), rRNA, snRNA, small nucleolar RNA (snoRNA), and tRNA obtained from the GenBank and Rfam 10.1 databases were then performed (Additional file 6: Table S5 and Additional file 7: Table S6). Table 1 shows the annotated of sRNAs. Among the unique sequences, 62,110 (0.46\%), 56,796 (0.44\%), 58,020 (0.45\%), and 60,639 $(0.42 \%)$ were identified to be similar to known miRNAs in RCK, RT, YACK, and YAT after searching the miRBase, respectively. Other types of unique sequences, including rRNA, snRNA, snoRNA, srpRNA, and tRNA were also detected in the four libraries. However, there were 13,132,245 (98.13\%), 12,686,933 (98.77\%), 12,816,045 (98.61\%), and 14,130,110 (98.89\%) unique sequences in RCK, RT, YACK, and YAT that could not be annotated, respectively, suggesting these sRNAs may be unique to sugarcane.

\section{Identification of known and novel miRNAs}

miRNA prediction was performed according to the formation and biometrics of miRNAs [60]. In the present study, the sequences of the miRNA precursors and mature miRNA in the four sugarcane samples in the unique sRNAs and miRBase databases were compared. BLAST analysis identified a total of 264, 263, 260, and 262 known miRNAs in RCK, RT, YACK, and YAT, respectively. The Mireap software was used to compare and screen the unannotated sequences (unann) using sugarcane ESTs from GenBank and transcriptome database of sugarcane in response to smut infection $(65,852$ unigenes) to predict novel miRNAs. Our results showed that there were 137, 140, 111, and 119 novel miRNAs in RCK, RT, YACK, and YAT, respectively. All of the miRNAs obtained by sequencing were mapped to the $S$. scitamineum genome database reported by Que et al. [14]. The result showed that none of the miRNA sequences was mapped to S. scitamineum 

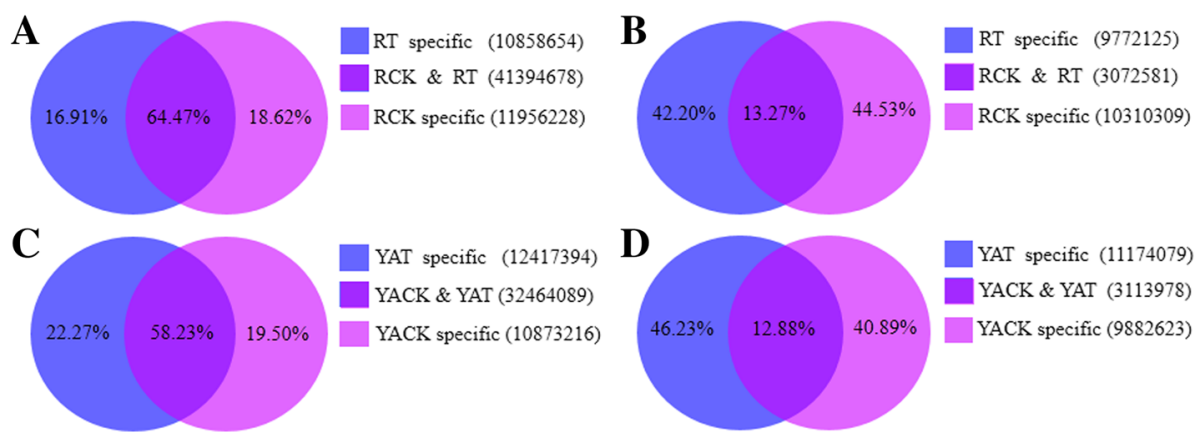

Fig. 1 The common and specific sRNAs in ROC22 and YA05-179 after Sporisorium scitamineum challenge. a Summary of total sRNAs between RT and RCK. $\mathbf{b}$ Summary of unique sRNAs between RT and RCK. $\mathbf{c}$ Summary of total sRNAs between YAT and YACK. $\mathbf{d}$ Summary of unique sRNAs between YAT and YACK. RCK and YACK: ROC22 and YA05-179 under sterile water stress after $48 \mathrm{~h}$, respectively; RT and YAT: ROC22 and YA05-179 under S. scitamineum stress after $48 \mathrm{~h}$, respectively

genome sequence, suggesting there was no contaminated S. scitamineum reads in the identified sugarcane miRNAs. Additional file 8: Figure S2A shows that the base distribution of the leading sites of novel miRNAs in the four samples were very similar. For example, the leading base of novel miRNAs of $20 \mathrm{nt}$ in length was $\mathrm{C}$, and majority of the leading base of novel miRNAs of 21 and 22 nt lengths was $\mathrm{U}$. The distribution and proportion of the four bases in the leading sites of novel miRNAs of $23 \mathrm{nt}$ in length were similar. The base distribution of novel miRNAs at different sites was identical in the four samples (Additional file 8: Figure S2B). In addition, the 11th nucleotide of the candidate miRNA sequences from all samples was generally $\mathrm{A}$.

\section{S. scitamineum-responsive miRNAs}

Transcriptional regulation in plants mainly relies on differentially expressed miRNAs [61]. In the present study, scatter plot analysis (Fig. 2a and b) showed that most differentially expressed known miRNAs in ROC22 and YA05-179 after S. scitamineum challenge was equally-expressed miRNAs. The number of upregulated miRNAs was less than that of downregulated
miRNAs. Under the screening criteria of $\log _{2}$-ratio $>1$ or $<-1$ and $P$-value $<0.01$, nine significantly upregulated and 26 significantly downregulated miRNAs (Additional file 9: Table S7) were detected among the 231 differentially expressed known miRNAs in the RT/RCK comparison; and nine significantly upregulated and two significantly downregulated miRNAs (Additional file 10: Table S8) were observed among the 208 differentially expressed known miRNAs in the YAT/YACK comparison. The value of differential fluctuation of differentially expressed known miRNAs in ROC22 and YA05-179 was up to -4.61- (miR5242) and -2.15 -fold (miR5152-3p), respectively.

The number of differentially expressed novel miRNAs was lower than that of known differentially expressed miRNAs. Most of the differentially expressed novel miRNAs were equally expressed miRNAs (Fig. 2c and d). RT/RCK comparison identified nine significantly upregulated and eight significantly downregulated miRNAs $(P$ value $<0.01)$ among the 48 differentially expressed novel miRNAs (Additional file 11: Table S9). In the YAT/YACK comparison, two significantly upregulated and four

Table 1 Annotation and classification of the small RNAs in the four libraries

\begin{tabular}{|c|c|c|c|c|c|c|c|c|}
\hline \multirow[t]{2}{*}{ Category } & \multicolumn{2}{|l|}{ RCK } & \multicolumn{2}{|l|}{ RT } & \multirow{2}{*}{$\begin{array}{l}\text { YACK } \\
\text { Unique }\end{array}$} & \multirow[b]{2}{*}{ Total } & \multicolumn{2}{|l|}{ YAT } \\
\hline & Unique & Total & Unique & Total & & & Unique & Total \\
\hline Total reads & $13,382,890$ & $36,396,588$ & $12,844,706$ & $27,812,972$ & $12,996,601$ & $27,464,468$ & $14,288,057$ & $28,290,231$ \\
\hline miRNA reads & 62,110 & $3,842,212$ & 56,796 & $2,769,304$ & 58,020 & $2,117,246$ & 60,639 & $1,973,307$ \\
\hline rRNA reads & 149,095 & $4,535,331$ & 80,458 & $1,347,015$ & 97,711 & $1,924,796$ & 76,758 & $1,102,526$ \\
\hline repeat reads & 526 & 1,214 & 489 & 1,114 & 424 & 1,067 & 443 & 1,153 \\
\hline snRNA reads & 6,390 & 25,149 & 4,072 & 11,579 & 4,800 & 12,774 & 4,897 & 12,309 \\
\hline snoRNA reads & 4,381 & 13,333 & 2,596 & 5,793 & 2,676 & 6,200 & 2,545 & 5,375 \\
\hline srpRNA reads & 2 & 2 & 0 & 0 & 0 & 0 & 0 & 0 \\
\hline tRNA reads & 28,141 & $1,578,262$ & 13,362 & 475,165 & 16,925 & 588,689 & 12,665 & 323,280 \\
\hline Unannotaed sRNA reads & $13,132,245$ & $26,401,085$ & $12,686,933$ & $23,203,004$ & $12,816,045$ & $22,813,696$ & $14,130,110$ & $24,870,281$ \\
\hline
\end{tabular}

RCK and YACK: ROC22 and YA05-179 under sterile water stress after $48 \mathrm{~h}$, respectively; RT and YAT: ROC22 and YA05-179 under Sporisorium scitamineum stress after $48 \mathrm{~h}$, respectively 

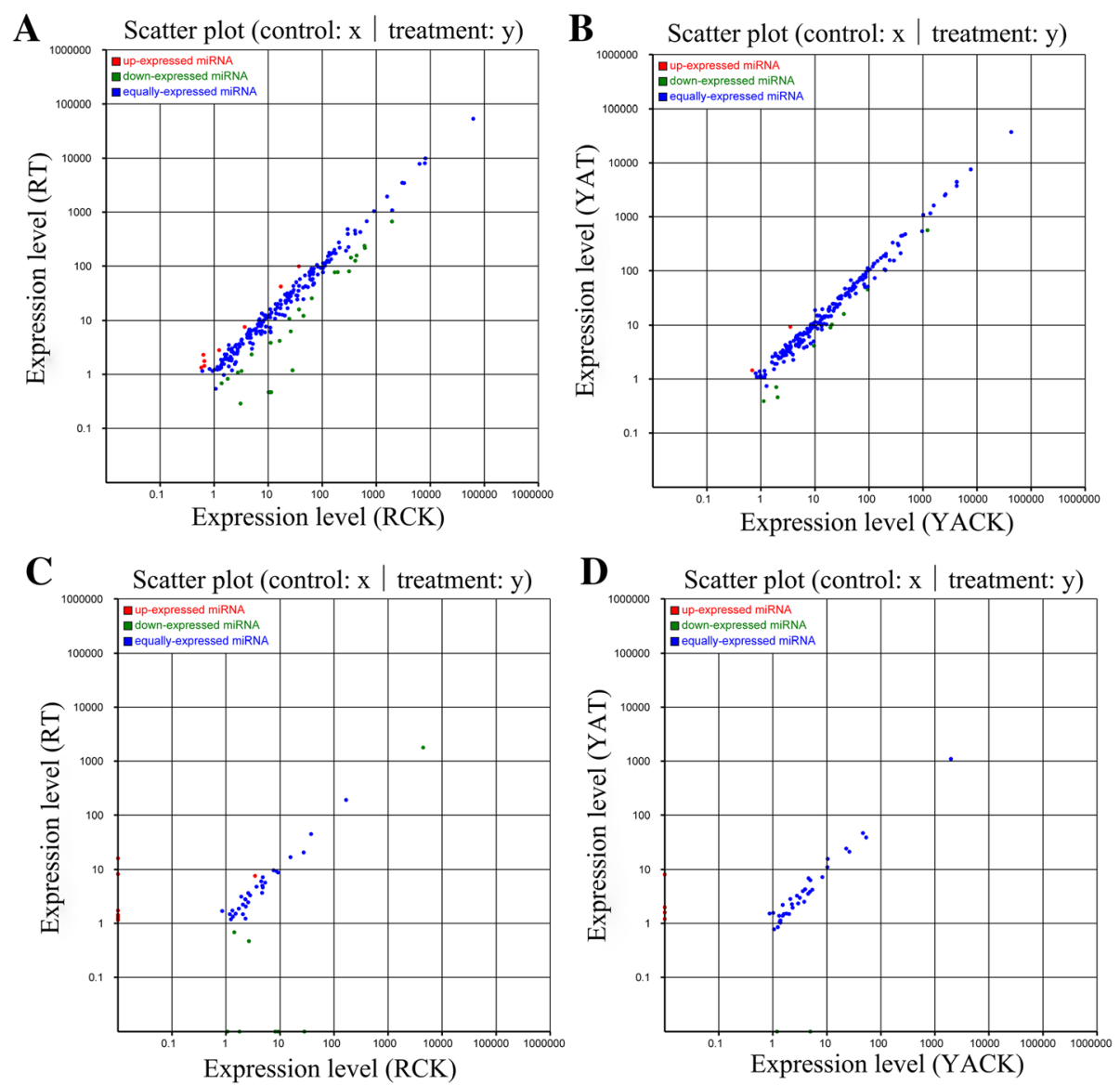

Fig. 2 Expression of miRNAs in ROC22 and YA05-179 after Sporisorium scitamineum challenge. a The differences of known miRNAs expression between RT and RCK. $\mathbf{b}$ The differences of known miRNAs expression between YAT and YACK. $\mathbf{c}$ The differences of novel miRNAs expression between RT and RCK. $\mathbf{d}$ The differences of novel miRNAs expression between YAT and YACK. The scatter plot of differentially expressed miRNAs (control: X-axis, treatment: $Y$-axis). The $X$ and $Y$ show the expression level of miRNAs in the two strains respectively. Red points mean miRNAs with $\log _{2}$-ratio $>1$; Blue points mean miRNAs with $-1 \leq \log _{2}$-ratio $\leq 1$; Green points mean miRNAs with $\log _{2}$-ratio $<1$. Ratio = normalized expression of the treatment/normalized expression of the control. RCK and YACK: ROC22 and YA05-179 under sterile water stress after $48 \mathrm{~h}$, respectively; RT and YAT: ROC22 and YA05-179 under S. scitamineum stress after $48 \mathrm{~h}$, respectively

significantly downregulated miRNAs $(P$-value $<0.01)$ were detected among the 41 differentially expressed novel miRNAs (Additional file 12: Table S10). Interestingly, the differentially expressed novel miRNAs with highly significant differential expression levels $(P$-value $<0.01)$ were specifically expressed in both sugarcane varieties. The differential fluctuation of differentially expressed novel miRNAs in ROC22 and YA05-179 was up to -11.46(novel_mir_11) and 9.65-fold (novel_mir_187), respectively.

Although differences in miRNA expression levels were significant, the expression trends (either up- or downregulated) of differentially expressed known miRNAs in both sugarcane varieties (RT/RCK and YAT/YACK) were generally similar (Fig. 3a). The specific regulatory modes of differentially expressed known miRNAs could be divided into four categories: significantly upregulated, upregulated in trace amounts, downregulated in trace amounts, and significantly downregulated. Most of the differentially expressed known miRNAs were categorized as significantly downregulated. Data analysis showed that the eight known miRNAs, miR1077-3p, miR262, miR5212-3p, miR5020a, miR1144a.1, miR5657, miR6149$3 p$, and miR536 were specifically expressed in YA05-179, but not in ROC22. Figure 3b shows that differentially expressed novel miRNAs were mostly detected in a single variety of sugarcane, but not in both sugarcane varieties.

\section{Prediction of target genes of miRNAs in sugarcane}

In the present study, the targeted genes of the known miRNAs, and novel miRNAs were subjected to prediction. The results indicated that most of the miRNAs had approximately ten target genes. Some miRNAs even had $>100$ target genes. According to the results of target gene prediction of known miRNAs (Additional file 13: Table S11), 32 differentially expressed known miRNAs of RT/RCK had 814 target genes, and ten differentially expressed known 


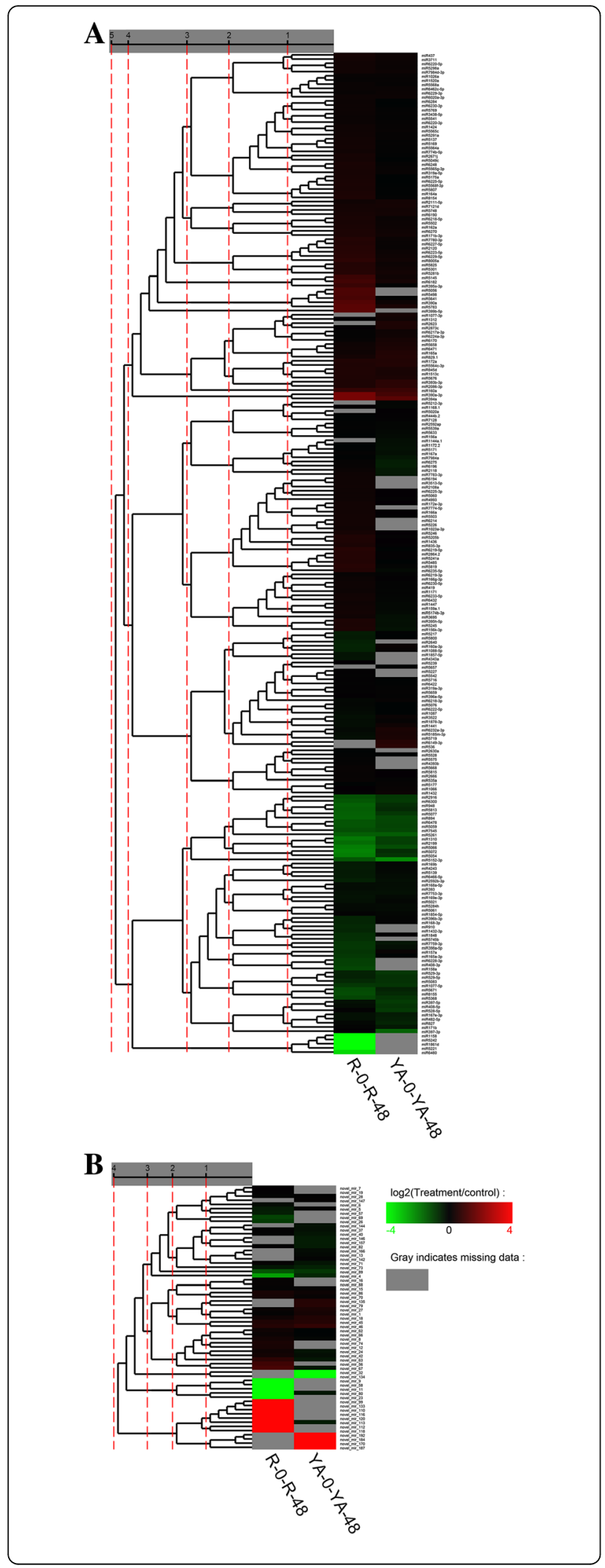

Fig. 3 Hierarchical clustering of differentially expressed known (a) and novel (b) miRNAs in ROC22 and YA05-179 after Sporisorium scitamineum challenge. R-0 and YA-0: ROC22 and YA05-179 under sterile water stress after $48 \mathrm{~h}$, respectively; R-48 and YA-48: ROC22 and YA05-179 under S. scitamineum stress after $48 \mathrm{~h}$, respectively

miRNAs of YAT/YACK had 127 target genes. Target gene prediction of novel miRNAs (Additional file 14: Table S12) showed that the 15 differentially expressed miRNAs of RT/ RCK had 457 target genes, and the six differentially expressed miRNAs of YAT/YACK were associated with 1,754 target genes. Additional file 14: Table S12 presents the prediction results for partial target genes of differentially expressed miRNAs. Analysis indicated that the function of these unannotated miRNA target genes were unknown or poorly characterized, thereby suggesting the possibility of new roles for these miRNAs in sugarcane in response to $S$. scitamineum.

\section{GO analysis of the predicted target genes}

GO enrichment analysis was conducted for the predicted target genes of differentially expressed miRNAs in RT/ RCK and YAT/YACK. Additional file 15: Figure S3 and Additional file 16: Figure S4 demonstrate the GO classification of the predicted target genes of known and novel miRNAs associated with biological processes, cellular components, and molecular functions. The main GO classification of target genes of differentially expressed known miRNAs and novel miRNAs in YAT/ YACK and RT/RCK was similar, which demonstrated that the differentially expressed miRNAs of the two sugarcane varieties after $S$. scitamineum challenge mainly targeted genes that were associated with cellular processes and metabolic processes. The predicted target genes were mainly associated with cell and organelle components. The molecular functions of the predicted target genes were mainly related to binding and catalytic activities.

\section{KEGG analysis of the predicted target genes}

KEGG pathway enrichment analysis identifies the most important physiological metabolic pathways and signal transduction pathways of candidate target genes [12]. In RT/RCK, the predicted target genes of known miRNAs had 13 pathways that were enriched $(P<0.05)$, and ten pathways that were significantly enriched $(P<0.01)$ (Additional file 17: Table S13). In YAT/YACK, the predicted target genes of known miRNAs had four pathways that were enriched $(P<0.05)$ and three pathways that were significantly enriched $(P<0.01)$ (Additional file 18 : Table S14), including pathogenic Escherichia coli infection, phagosome, and other types of O-glycan biosynthesis. In RT/RCK, the predicted target genes of novel 
miRNAs had 13 pathways that were enriched $(P<0.05)$ and 11 pathways that were significantly enriched $(P<0.01)$ (Additional file 19: Table S15). In YAT/YACK, the predicted target genes of novel miRNAs showed 13 pathways that were enriched $(P<0.05)$ and five pathways that were significantly enriched $(P<0.01)$ (Additional file 20: Table S16).

The significantly enriched KEGG pathway of the predicted target genes in sugarcane after $S$. scitamineum challenge (Fig. 4) could be divided into five types, including stress response pathway (i.e., plant-pathogen interaction, apoptosis, pathogenic Escherichia coli infection, phagosome, cutin, suberine and wax biosynthesis, and peroxisome), hormone and signal transduction pathways (i.e., calcium signaling pathway, MAPK signaling pathway, plant hormone signal transduction, zeatin biosynthesis, and brassinosteroid biosynthesis), metabolic pathway (i.e., stilbenoid, diarylheptanoid and gingerol biosynthesis, polycyclic aromatic hydrocarbon degradation, phenylalanine metabolism, bisphenol degradation, glucosinolate biosynthesis, other types of O-glycan biosynthesis, N-Glycan biosynthesis, and pantothenate and CoA biosynthesis), transcription and protein synthesis pathways (i.e., RNA polymerase, RNA degradation, mRNA surveillance pathway, RNA transport, and aminoacyl-tRNA biosynthesis), and cell division pathway (i.e., cell cycle, DNA replication, meiosis, nucleotide excision repair, homologous recombination, mismatch repair, base excision repair, and non-homologous end-joining). In general, miRNA target gene enriched pathways of both sugarcane varieties were mostly identical, but not in the number of target genes and the degree of significant enrichment in the pathways.

\section{Validation of miRNAs by qRT-PCR analysis}

In the present study, 12 candidate differentially expressed known miRNAs with different expression levels, including miR394a, miR408-3p, miR397-3p, miR7545, miR5066, miR5261, miR948, miR5059, miR5783, miR5077, miR6300, and miR894, as well as four candidate differentially expressed novel miRNAs, including novel_mir_133, novel_mir_99, novel_mir_32, and novel_mir_58 were screened and further analyzed via qRT-PCR to verify their expression. Among the 16 miRNAs, miR408-3p, novel_mir_133, novel_mir_99, novel_mir_32, and novel_mir_58 were specifically expressed in a single sugarcane variety. As shown in Fig. 5, except for four miRNAs, including miR5261 and miR397-3p in YA05-179, as well as miR5077 and novel_mir_99 in ROC22, the miRNA expression patterns (up- or downregulated) as measured by quantitative analysis and the sequencing results of the miRNAs were generally similar.

In addition, we performed qRT-PCR analysis of the 12 candidate differentially expressed miRNAs at different time points $(0,12,48$, and $96 \mathrm{~h})$ after $S$. scitamineum

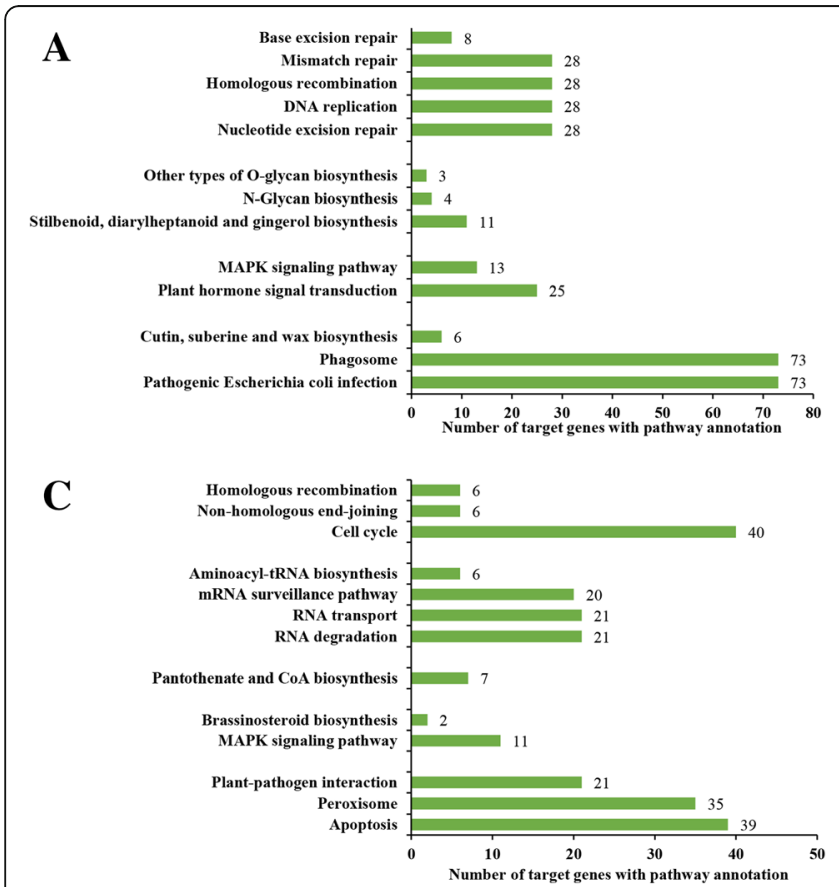

\section{B}
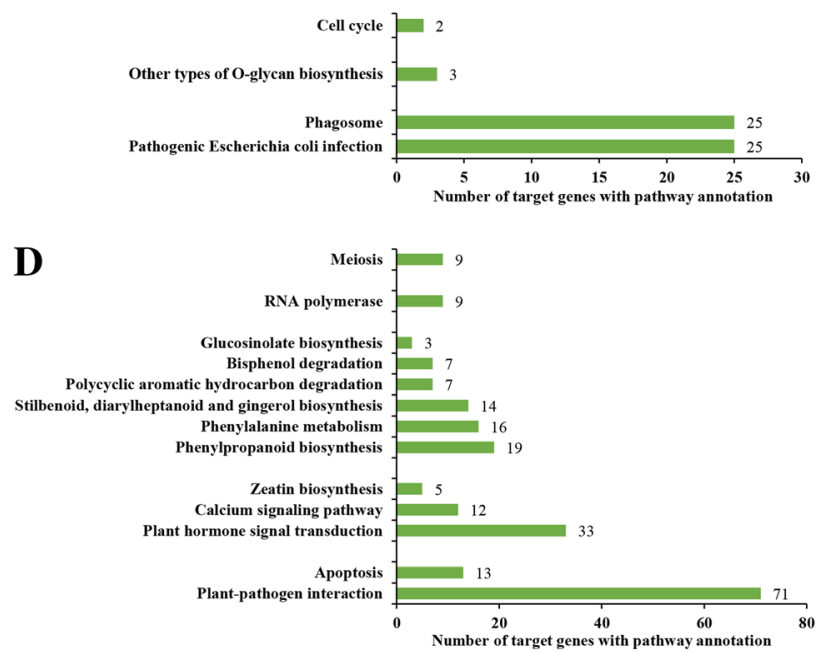

Fig. 4 The significantly enriched KEGG pathway ( $P$-value $<0.05)$ of the predicted target genes. The genes were targeted by differently expressed known and novel miRNAs in RT/RCK (a and $\mathbf{c}$ ) and YAT/YACK (b and $\mathbf{d}$ ), respectively. RCK and YACK: ROC22 and YA05-179 under sterile water stress after $48 \mathrm{~h}$, respectively; RT and YAT: ROC22 and YA05-179 under S. scitamineum stress after 48 h, respectively. Number of target genes with pathway annotation was shown in the bar charts 


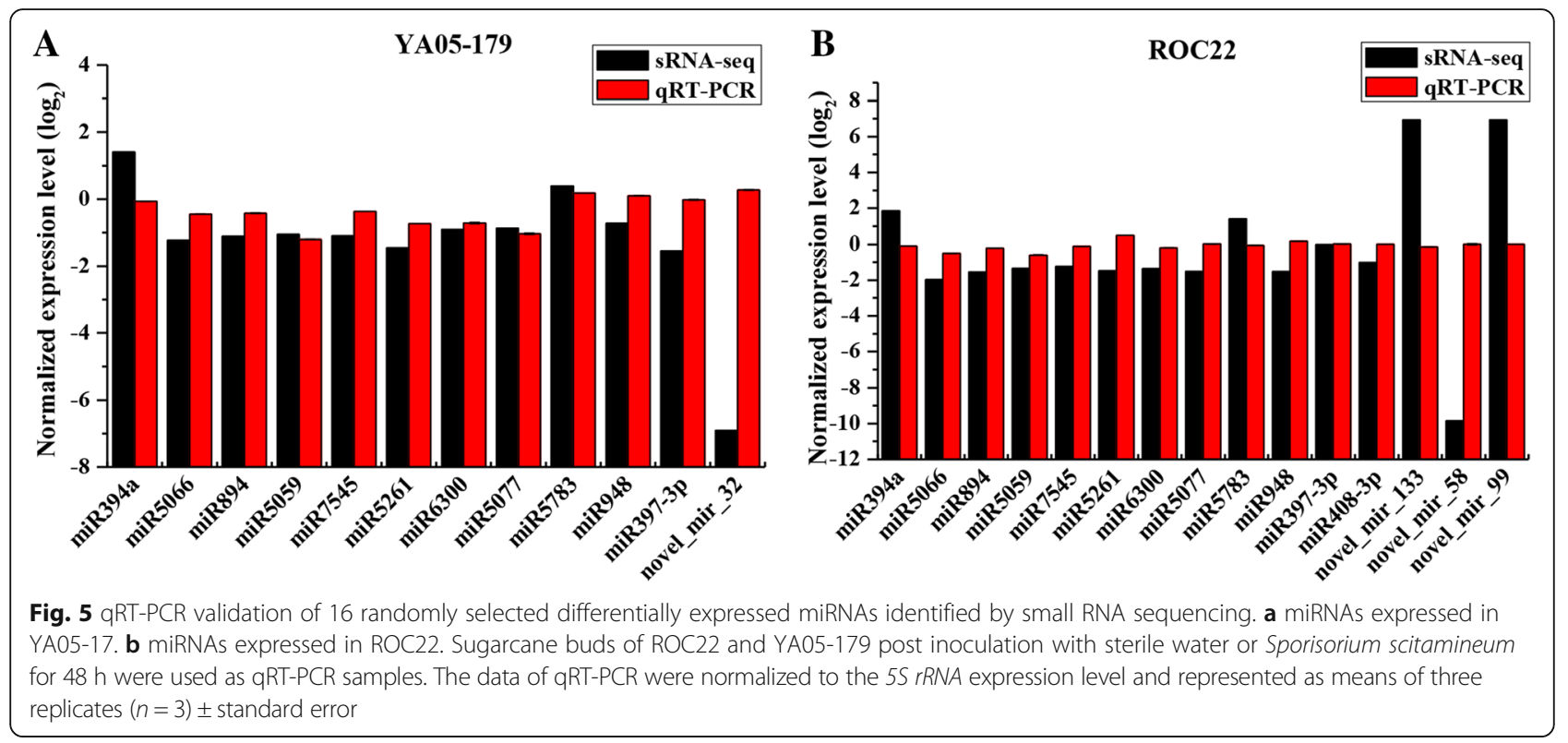

challenge. As shown in the line charts of Fig. 6, seven miRNAs, including miR394a, miR7545, miR894, miR397-3p, miR5261, miR5783, and miR948 were determined to be expressed in both sugarcane varieties, YA05-179 and ROC22. A specific expression pattern for miR5261 was observed, which involved a drastic upregulation in the sugarcane smut-resistant genotype, YA05-179, after inoculation of S. scitamineum. Its expression reached 1,000-fold at $48 \mathrm{~h}$ after S. scitamineum challenge and declined to 0 -fold at $96 \mathrm{~h}$. On the other hand, in the smut-susceptible variety, ROC22, miR5261 expression drastically increased after $S$. scitamineum
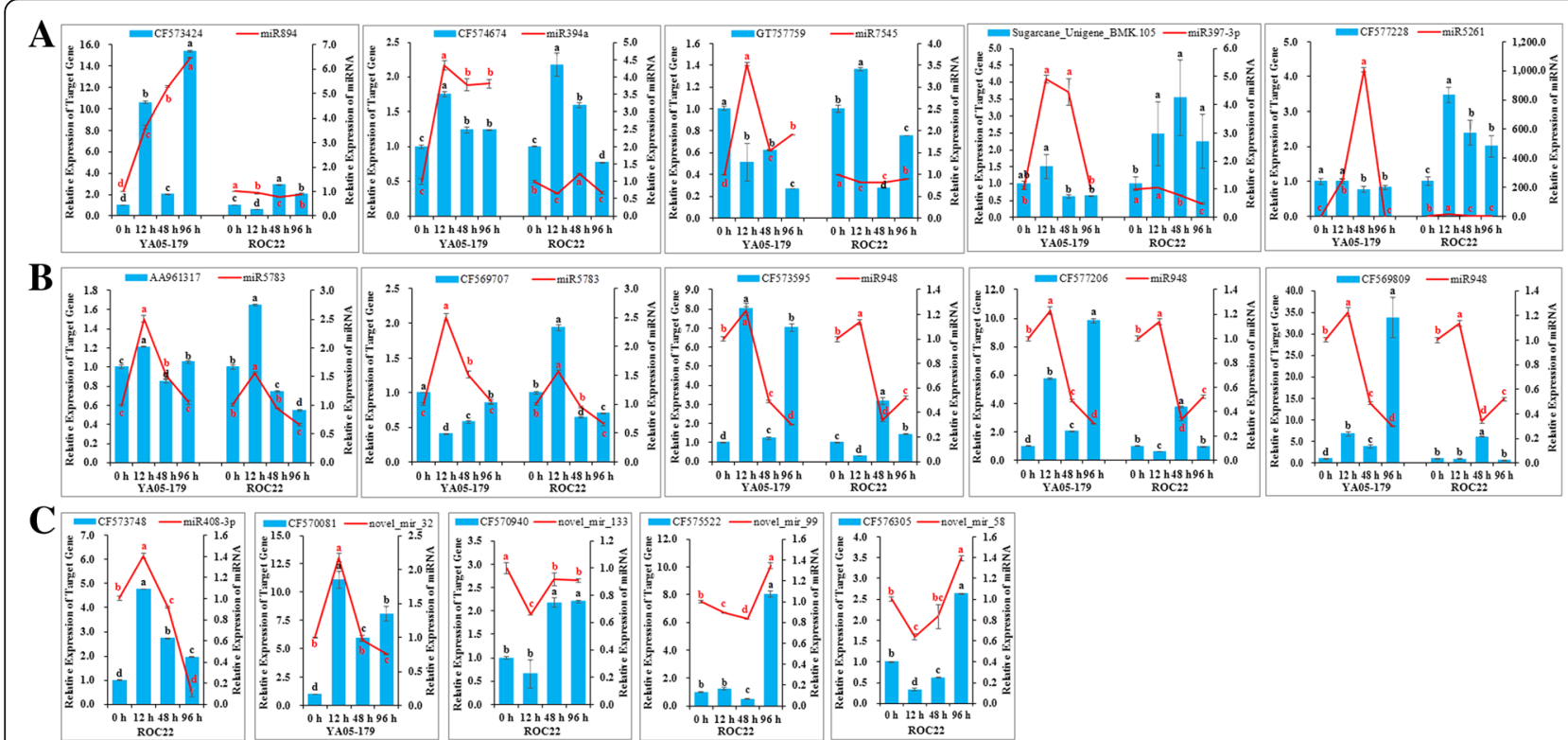

Fig. 6 Expression patterns of selected 12 miRNAs (line charts) and 15 predicted target genes (bar charts) in ROC22 and YA05-179 at 0, 12, 48, and $96 \mathrm{~h}$ after Sporisorium scitamineum challenge. Gene expression levels were assessed by qRT-PCR. The right and left y axis represented the relative expression of miRNA and its predicted target gene, respectively. The relative expression levels of miRNAs and their predicted target genes were normalized to the $5 S$ rRNA and glyceraldehyde-3-phosphate dehydrogenase (GAPDH) expression levels, respectively. Each bar represented as means of three replicates $(n=3) \pm$ standard error. Different lowercase letters indicate a significant difference, as determined by Duncan's new multiple range test ( $P$-value $<0.05)$. The names of miRNA and corresponding targeted gene were listed in the top of each panel. Five out of twelve miRNAs, including miR408-3p, novel_mir_32, novel_mir_133, novel_mir_99, and novel_mir_58, were expressed specifically in ROC22 or YA05-179 after S.

scitamineum challenge 
challenge and reached 11 -fold at $12 \mathrm{~h}$ post-inoculation, followed by a decline at $48 \mathrm{~h}$ and remained unchanged at $96 \mathrm{~h}$. Among the seven miRNAs, the expression patterns of six miRNAs, except for miR5261, could be generally divided into two types: first, opposite expression patterns in YA05-179 and ROC22 (Fig. 6a), and second, identical expression pattern in YA05-179 and ROC22, which referred to similar relative expression regulatory trends of miRNAs in the two sugarcane varieties and at different treatment time points after $S$. scitamineum challenge (Fig. 6b). There were four miRNAs, which included miR394a, miR894, miR7545, and miR397-3p that demonstrated the first type of expression pattern, whereas miRNAs involved in the second expression pattern consisted of miR948 and miR5783. However, miR5783 was upregulated in YA05-179, but upregulated and subsequently downregulated in ROC22. On the other hand, both miR948 and miR5783 were upregulated within $12 \mathrm{~h}$ after $S$. scitamineum challenge, which was followed by its downregulation. In addition, the remaining five miRNAs (i.e., miR408-3p, novel_mir_133, novel_mir_99, novel_mir_58, and novel_mir_32) were only expressed in a single variety (Fig. 6c). Among these, miR408-3p, novel_mir_133, novel_mir_99, and novel_mir_58 were specifically expressed in ROC22. miR408$3 p$ was markedly increased at $12 \mathrm{~h}$ and subsequently downregulated from 48 to $96 \mathrm{~h}$ after S. scitamineum challenge. Novel_mir_133 was downregulated at 12, 48, and $96 \mathrm{~h}$ compared to control. Novel_mir_99 and novel_mir_58 showing an overall downregulation, followed by a slight upregulation at 12 and 96 h. Novel_mir_32 in YA05-179, demonstrated an "upregulateddownregulated" specific expression pattern.

\section{Validation of the predicted target genes by qRT-PCR analysis}

qRT-PCR analysis of 15 predicted target genes of differentially expressed known and novel miRNAs in different sugarcane varieties was conducted (Fig. 6) at different time points (i.e., 0-96 h) after S. scitamineum challenge. Despite the specific expression of predicted target gene CF573748 of differentially expressed known miRNA miR408-3p (Fig. 6c), the predicted target genes (i.e., CF570081, CF570940, CF575522, and CF576305) of four novel miRNAs (Fig. 6c) were detected in a single sugarcane variety, and the remaining predicted target genes were expressed in both sugarcane varieties (Fig. 6a and b).

Figure 6 shows the results of 11 predicted target genes of eight candidate differentially expressed known miRNAs. The expression of predicted target gene CF573748 in ROC22 was generally upregulated. The other predicted target genes were divided into two categories based on its expression patterns: (i) Significantly upregulated expression in ROC22, which was mostly higher than that in the upregulated expression in YA05-179. The observed upregulation in YA05-179 was gradual or mostly downregulated at $12 \mathrm{~h}$ after $S$. scitamineum challenge compared to that at $0 \mathrm{~h}$ of $\mathrm{S}$. scitamineum challenge. This category included CF574674, Sugarcane_Unigene_BMK.105, CF577228, AA961317, CF569707, and GT757759. (ii) Significantly and generally upregulated expression in YA05-179 compared to that at $0 \mathrm{~h}$ of $S$. scitamineum challenge, whereas it was first downregulated, followed by moderate upregulation in ROC22 after S. scitamineum challenge. This category included CF573424, CF573595, CF577206, and CF569809. Figure $6 \mathrm{c}$ shows that the overall expression of the predicted target gene, CF570081, of novel miRNA novel_mir_32 in YA05-179 was upregulated, whereas that of the three predicted target genes, i.e., CF570940, CF575522, and CF576305 in ROC22 was initially downregulated, and subsequently upregulated with an extended duration of $S$. scitamineum challenge.

\section{Discussion \\ Identification and annotation of miRNAs and high- throughput sequencing of sugarcane sRNAs}

YA05-179 is a BC4 generation of sugarcane and Eranthus arundinacus with high smut-resistance, whereas ROC22 is a highly smut-susceptible variety. These two sugarcane varieties have completely opposite disease-resistance performance and thus are good models for gene regulation during sugarcane smut infection. A previous study has shown that under natural stress, the disease symptoms of sugarcane smut usually occur two to four months after the dissemination of pathogens [62], with the phenocritical period significantly later than the gene regulation period. To maintain a consistent physiological and biochemical condition, the present study used the artificial inoculation with $5 \times 10^{6}$ spores $/ \mathrm{mL}$ smut spore suspension [63]. Su et al. [8] showed that the logarithmic growth period of the pathogens was at $48 \mathrm{~h}$ after the artificial inoculation of sugarcane smut pathogens, and the stationary phase was at $96 \mathrm{~h}$ post-inoculation, suggesting that the critical period for physiological and biochemical reactions in sugarcane in response to the smut disease was the first $48 \mathrm{~h}$ after inoculation.

Solexa sequencing technology, HiSeq sequencing system, 454 sequencing, and sequencing by oligonucleotide ligation and detection (SOLiD) sequencing technology [64], which are relatively mature and optimized and have been widely used in the analysis of plant miRNA expression profiles [65], the screening of differentially expressed miRNAs under biotic and abiotic stresses [66], the identification of miRNAs associated with growth and development [67], and the discovery of novel miRNAs with new functions [68]. In the present study, we used HiSeq sequencing system to identify differentially expressed miRNAs in sugarcane after challenging with the pathogen 
for smut disease. The number of clean reads obtained from each RCK, RT, YACK, and YAT contained $<1 \%$ of contaminated sequences and therefore, $99 \%$ of the sequences were clean reads. Statistical data of analyses of the sequence length and the common and specific sequences showed that the sequencing quality of each sample was relatively high, with good overall consistency among different samples.

The genetic background of sugarcane is complicated, and genome analysis of sugarcane is currently in its preliminary stages [69]. ESTs are only a part of the annotation results in the database, suggesting that most of the unknown sequences have not been characterized. Sequences detected from the four sugarcane samples were not directly matched with the miRNA sequences in the miRBase database. For the prediction of novel miRNAs, we combined our sequencing data with the Sugarcane_Unigene sequences of the sugarcane transcriptome database, which was provided by our laboratory. The sequencing results of the present study were comparable to the entries in the sugarcane transcriptome database [47] and the sugarcane EST in GenBank, thereby indicating that our findings were reliable. Statistical analysis of base distribution of novel miRNAs demonstrated that the most common base of novel miRNAs of 21 and $22 \mathrm{nt}$ in length was $U$. In addition, nucleotide 11 of candidate miRNA sequences of the four samples was A. A previous study revealed that plant miRNAs mostly act via the degradation of target mRNAs [70]. The cleavage of this degradation occurred very precisely at nucleotide 10 or 11 of the matched miRNAs [70]. In addition, miRNAs bound to the argonaut (AGO) protein later complementarily bound to the mRNA 3' untranslated region of the target gene, thereby cleaving or degrading the target gene [71]. In the present study, the $5^{\prime}$ end of 21- and 22-nt novel miRNA sequences preferentially harbored the $U$ base, indicating that these novel miRNA sequences might play an important role in regulating gene expression after smut pathogen challenge. In addition, nucleotide 11 of candidate miRNA sequences in the four samples tended to be A, which was consistent with the bias of cleavage sites of degradation, as well as the statistical results of the base, thereby suggesting that the prediction method used in the present study was feasible. The above results showed that the quality of the measured data was high, thus meeting the requirements for subsequent analysis.

\section{Screening and analysis of differentially expressed miRNAs by using QRT-PCR}

High-throughput sequencing generates massive data on miRNAs. Therefore, the present study exclusively focused on screening of differentially expressed miRNAs after $S$. scitamineum challenge. The present study identified individual differences in miRNA expression in the two sugarcane varieties, YA05-179 and ROC22, after the challenge of sugarcane smut pathogen, as well as differences between the two varieties. In terms of individual differences of each variety after S. scitamineum challenge, ROC22 had 231 differentially expressed miRNAs, which included 35 significantly expressed miRNAs (| $\log _{2}$-ratio| $>1, P<0.01$ ), and the read count of miR2199, miR2916, miR5077, miR5813, miR6300, and miR894 was $>10,000$. Among the 208 differentially expressed miRNAs screened in YA05-179, 11 miRNAs showed significant individual differences in miRNA expression, of which the read count of miR894 was also $>10,000$. In terms of differences between two sugarcane varieties, when ROC22 and YA05179 were subjected to smut disease, the differentially expressed miRNAs screened from the individual known miRNAs were mostly the same. However, miR397-3p (-1.54-fold) was upregulated in YA05-179 only, although not very high. Eight known miRNAs, including miR10773p, miR262, miR5212-3p, miR5020a, miR1144a.1, miR5657, miR6149-3p, and miR536, were specifically expressed in the smut resistant-genotype, YA05-179. Cluster analysis of expression patterns of known miRNAs in the two sugarcane varieties showed that most miRNAs changed in the same way in the two varieties following treatment (either upregulated in both or downregulated in both), with only differences found in the degree of the changes. Some regulatory modes of the miRNAs were inconsistent, although not significant. The present study identified 48 novel miRNAs that were differentially expressed in ROC22, of which 16 were upregulated. Forty-one differentially expressed novel miRNAs were detected in YA05-179, of which six were upregulated. The differentially expressed miRNAs with significant expression levels in both control and treated groups were almost specifically expressed in a single sugarcane variety, with differentially expressed novel miRNAs determined to be the majority. No novel miRNA was differentially expressed in both sugarcane varieties. In addition, to ensure the reliability of expression profiling data, miRNAs obtained from high-throughput sequencing should be verified in plant tissues, commonly by qRT-PCR [72]. The present study validated the 27 expression profiles correspond to 16 differentially expressed miRNAs in ROC22 and YA05-179 (Fig. 5). Except for the unmatched expression level and sequencing results of miR5261 and miR397-3p in YA05-179 and miR5077 and novel_mir_99 in ROC22, the expression patterns (up- or downregulated) of the remaining miRNAs and their corresponding sequencing results were consistent, suggesting that the sequencing results of the present study were reliable. Whereas the minor discrepancy between the qRT-PCR expression levels and sequencing results may possibly be due to different software and algorithms used in processing the vast amount of data generated from high-throughput 
sequencing $[73,74]$. Overall, the results indicated that the high-throughput sequencing was a powerful tool for discovering novel and differentially expressed miRNAs in sugarcane after $S$. scitamineum challenge, and it is also anticipated that more replicates in further work should help to ensure the accuracy of the sequencing results.

Previous studies have demonstrated that the regulatory networks of the target genes of plant miRNAs in response to the environmental stress are centrally located, thereby affecting a variety of regulatory signals $[67,75]$. In the present study, we focused on the possible regulatory roles of differentially expressed known and novel miRNAs in sugarcane after smut pathogen challenge. Seven miRNAs, including miR894, miR394a, miR7545, miR397-3p, miR5261, miR5783, and miR948, were coexpressed in both sugarcane varieties after the inoculation of S. scitamineum and showed different expression patterns. This study also identified a specifically expressed miRNA, namely, miR5261, in YA05-179 and ROC22, with highly significant fold changes in the expression and a relatively wide range of differential fluctuations in expression levels. Five miRNAs, including miR408-3p, novel_mir_32, novel_mir_133, novel_mir_99, and novel_mir_58 were only detected in a single sugarcane variety after S. scitamineum inoculation. However, the specific roles of differentially expressed miRNAs on the target genes in resisting S. scitamineum after sugarcane smut infection remain unclear and need to be further investigated.

\section{Prediction and expression analysis of target genes of differentially expressed miRNAs}

Sugarcane is a highly heterozygous allopolyploid and aneuploid crop [69]. The present study performed a comprehensive analysis of existing transcriptome sequences and our experimental sequencing results. However, because whole-genome sequencing of sugarcane has not been completed [69], majority of the sRNA sequences during miRNA annotation remained unknown. The results of the present study indicated that although the number of predicted target genes of the whole miRNAs in the YA05-179 and ROC22 varieties was relatively equivalent, that of differentially expressed known miRNAs in YA05-179 (127) was less than that observed in ROC22 (814). On the other hand, the predicted target gene number of differentially expressed novel miRNAs in YA05-179 (1754) was significantly higher than that in ROC22 (457). The number of differentially expressed known miRNAs in YA05-179 (10) after S. scitamineum challenge was slightly lower than that in ROC22 (32). Predictive analysis of differentially expressed novel miRNA candidates in both sugarcane varieties demonstrated that only a few novel miRNA candidates were co-expressed in both sugarcane varieties. On the other hand, most of the novel miRNA candidates were specifically expressed in a single variety and majority of these were upregulated. Prediction results of the above target genes indicated that novel miRNAs screened from YA05-179 were more significant in the evaluation of the molecular mechanism underlying the interaction between sugarcane and S. scitamineum.

Functional analysis of target genes of miRNAs is the most direct approach in studying the function of miRNAs [20]. Bioinformatics analysis can effectively predict the target genes of miRNAs, including its functions [76]. In the present study, we focused on identifying the regulatory role of differentially expressed miRNAs, with functional analysis and annotation of potential target genes from the GO and KEGG pathways. In the three GO categories, namely, biological processes, cellular components, and molecular functions, the classifications of predicted target genes of the differentially expressed novel miRNAs in both sugarcane varieties were similar to the sequencing data on the predicted target genes of differentially expressed known miRNAs. The target gene distribution in the biological processes was mainly associated with cellular processes and metabolic processes. KEGG pathway enrichment analysis demonstrated that the predicted target genes of differentially expressed miRNAs participated in a series of biochemical pathways or disease resistance-related physiological, metabolic, and signal transduction pathways such as plant-pathogen interaction, peroxisome, apoptosis, phagosome, cutin, suberine and wax biosynthesis, plant hormone signal transduction, MAPK signaling pathway, calcium signaling pathway, zeatin biosynthesis, and brassinosteroid biosynthesis. Although the predicted target genes of the identified differentially expressed miRNAs from different sugarcane varieties were not the same, its response processes almost covered every aspect throughout the life course, and its metabolic regulatory pathways were identical. Previous studies have also shown that the molecular mechanism of the interaction between sugarcane and $S$. scitamineum was regulated by multigenic network systems, and the pathogen of sugarcane smut also activated a variety of smut-resistance metabolic pathways [77, 78]. In addition, our findings prompted us to speculate that miRNAs post-transcriptionally regulate mRNAs, which are consistent with the results of previous studies [77, 78]. Upregulation of miRNAs may result in the degradation of target genes or the downregulation of miRNAs may promote the overexpression of target genes, thereby changing a number of metabolic or signal transduction pathways.

\section{Expression analysis of several predicted target genes using qRT-PCR}

Prediction and functional analysis of the target genes of differentially expressed miRNAs are efficient approaches in studying the functions of miRNAs [20]. In the present study, we used qRT-PCR to evaluate the correlation 
between miRNAs and its predicted target genes and analyzed the expression patterns of the target genes of differentially expressed miRNAs in the same sugarcane variety at different time points after S. scitamineum inoculation. In total, 15 predicted target genes of 12 miRNAs were detected, including 11 predicted target genes of eight known miRNAs and four predicted target genes of four novel miRNAs (Fig. 6). We combined the miRNA expression patterns and the target gene expression patterns to analyze the quantitative expression of target genes at four different time points and its corresponding miRNA expression.

qRT-PCR analysis (Fig. 6) showed that except for the inconsistent expression between the predicted target gene, CF569707, and its corresponding miRNA, miR5783, ten out of 11 predicted target genes of known miRNAs had basically identical negatively regulated mode after $12 \mathrm{~h}$ and reached the highest degree of matching at $48 \mathrm{~h}$. In particular, the expression patterns of three corresponding target genes of miR948 were identical, suggesting that the expression of these genes was highly consistent with that of the miRNAs. These results also indicated that the regulatory effect of the corresponding genes of miRNAs after S. scitamineum challenge was maximized at $48 \mathrm{~h}$ after postinoculation. However, this is a negative regulatory effect. The above results also showed that the expression enrichment prediction of known miRNAs and its predicted target genes in the present study was reliable. Our prediction of biochemical, metabolic, or signal transduction pathways associated with the miRNAs and its predicted target genes might provide novel insights on future anti-smut research studies. In addition, the negatively regulated role in quantitative expression between the novel miRNAs and its predicted target genes was not extremely high. The expression of CF570081 and CF57094 was anti-correlated with miRNA results at 48 and $96 \mathrm{~h}$ but the expression of CF575522 and CF576305 was not. It is possible that these potential target genes are regulated by more than one miRNAs at the translational level [79]. Meanwhile, due to the lacking of relevant research and also the lack of sugarcane genomic information, many of the novel miRNAs identified in the present study are being reported for the first time. These miRNAs do represent a portion of novel miRNAs involved with smut pathogen challenge, however it needs more samples or sequencing coverage to extract more reliable and functional novel miRNAs. Further improvement in methods and strategies may be necessary for the prediction and functional analysis of novel miRNAs, such as mismatches of nucleotide sequences of miRNA, which may cause errors in gene targeting [60].

\section{Analyses of predicted target gene functions and smut resistance-related metabolic pathways}

A previous study demonstrated that in response to pathogen infection, the appearance, physiological, and biochemical changes in host cultivars are ultimately caused by the disruptions at the molecular level [80]. Diseasesusceptible varieties show relatively slow and weak responses and signals to infection, whereas the responses of signals of disease-resistant varieties are relatively rapid and strong [80]. For these reasons, the disease-resistant plant varieties could combat most of the damages inflicted by the pathogen, as well as prevent its proliferation and further spread [80]. In the present study, the predicted target genes of differentially expressed miRNAs in ROC22 and YA05179 were determined to participate in several metabolic pathways after S. scitamineum challenge. Notably, three possible pathways associated with smut pathogen stress, including plant-pathogen interaction pathway, MAPK signalling pathway, and plant hormone signal transduction, were chosen for further analysis. The expression profiles of five crucial miRNAs and eight of their predicted target genes in YA05-179 and ROC22 after S. scitamineum challenge for $48 \mathrm{~h}$ were confirmed by qRT-PCR (Fig. 7).

\section{MAPK signaling pathway in plants}

When plant cells are exposed to environmental stress and receive hormonal signals, MAPK cascade pathway was activated to induce the expression of specific transcription factors in the nucleus and functional gene that would, in turn, activate other protein kinases in the cytoplasm to ultimately trigger the plant cell to elicit physiological and biochemical responses [81]. A previous study has shown that when rice crops were infected by Pyricularia grisea, several genes belonging to the MAPK pathway were upregulated [82]. In the present study, the predicted target genes of some miRNAs participated in the MAPK cascade pathway. As reported, expression of the protein kinase A $(P K A)$ gene significantly inhibits the activities of several members of the MAPK family, thereby blocking the MAPK signaling pathway [83]. Wheareas, the target PKA gene (CA133877) of miR5783 was significantly upregulated (1.39-) in the smut-resistant genotype YA05-179, but downregulated (0.44-) in the smut-susceptible genotype ROC22 (Fig. 7). Similarly, miR5783 was also significantly upregulated (1.52-) in YA05-179 and downregulated (0.95-) in ROC22 but not at significant level. We speculated that miR5783 might positively regulate PKA expression, thereby regulating related genes in the MAPK pathway. Heat shock protein 72 (HSP72) is a chaperone protein that plays an important regulatory role in the cells of various organisms [84]. During adverse conditions such as high temperature, endotoxin, and oxidative stress, organisms stimulate and induce the synthesis of HSP72 [84]. In the present study, miR5221 was significantly downregulated (0.51-) in YA05-179 and slightly upregulated (1.11-) in ROC22 which was not significant. Expression level of the predicted target gene of miR5221, HSP72 (Sugarcane_Unigene_BMK.31740), was significantly increased 


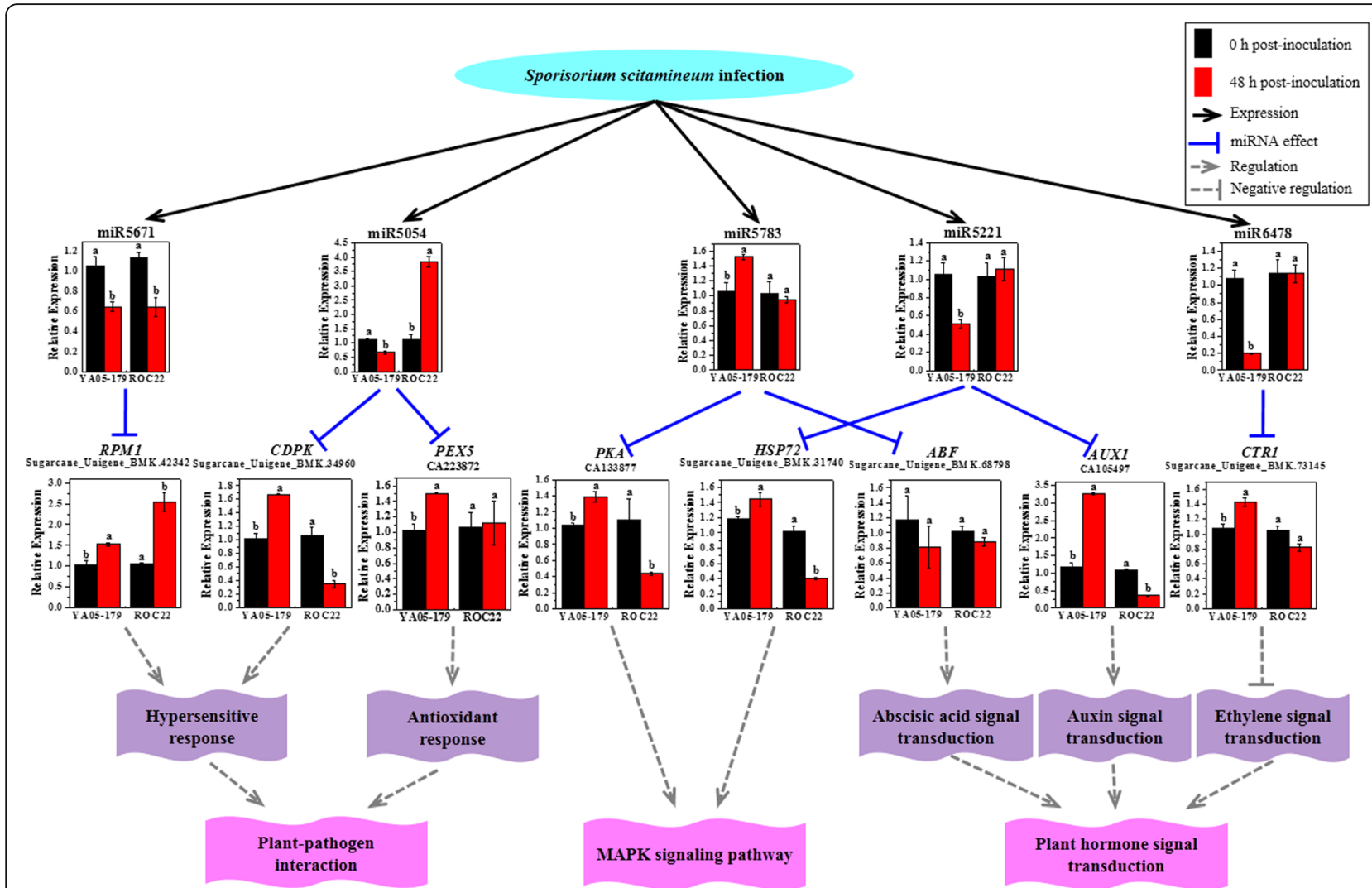

Fig. 7 A proposed regulatory network of partial miRNAs in sugarcane after Sporisorium scitamineum challenge. The gene expression profiles of five differentially expressed miRNAs, including miR5671, miR5054, miR5783, miR5221, and miR6478, as well as eight of their target genes, including RPM1 (Sugarcane_Unigene_BMK.42342), CDPK (Sugarcane_Unigene_BMK.34960), PEX5 (CA223872), PKA (CA133877), HSP72 (Sugarcane_Unigene_BMK.31740), ABF (Sugarcane_Unigene_BMK.68798), AUX1 (CA105497), and CTR1 (Sugarcane_Unigene_BMK.73145), were validated by qRT-PCR. The sugarcane buds from ROC22 and YA05-179 inoculated with distilled water and S. scitamineum at 0 and $48 \mathrm{~h}$ were used for qRT-PCR samples. The expression profiles of the miRNAs were normalized to the 5S rRNA expression level and represented as means of three replicates $(n=3) \pm$ standard error. The expression profiles of the target genes were normalized to the glyceraldehyde-3phosphate dehydrogenase (GAPDH) expression level and represented as means of three replicates $(n=3) \pm$ standard error. Different lowercase letters indicate a significant difference, as determined by Duncan's new multiple range test ( $P$-value $<0.05)$. RPM1, effector-triggered immune receptor; $C D P K$, calmodulin-independent protein kinase; PEX5, peroxisome 5; PKA, protein kinase A; HSP72, heat shock protein 72; ABF, ABA-responsive element-binding protein; AUX1, auxin transporter; CTR1, constitutive triple-response 1

(1.44-) in YA05-179 and significantly decreased (0.40-) in ROC22, suggesting that miR5221 may play a negative regulation role in $H S P 72$ expression to combat smut infestation.

\section{The signal transduction pathways of plant hormones}

Plant hormones play an important role in the response to environmental stresses [85]. The most common plant hormones include auxin (namely indole-3-acetic acid, IAA), cytokinin (CK), abscisic acid (ABA), gibberellin acid (GA), ethylene (ET), brassinosteroid (BR), salicylic acid (SA), jasmonic acid (JA), and polyamines. A previous study has shown that plant hormones play a critical role in plant defenses against pathogens [86]. During growth and development, plants generate regulatory defense responses to abiotic stresses, which include the endogenous hormones, SA, ET, and JA. During abiotic stress in plants, ABA interacts with the SA, JA, and ET-regulated signal transduction pathways and negatively regulates the resistance of abiotic stress in plants [87]. IAA induces the transient expression of some genes in response to abiotic stress [88]. AUX1 (auxin transporter) is an auxin influx carrier protein that plays a role in cellular transport in the plant root system [89]. Overexpression of $A U X 1$ accelerates the transportation of IAA, which regulates the expression of a stress-related gene, $\mathrm{C}$-repeat binding factor (CBF) [90]. In the present study, the predicted target genes of the differentially expressed miRNAs participate in a variety of hormone signaling pathways, thereby suggesting that these miRNA-regulated target genes play critical roles in signal transduction pathways involving plant hormones. Among these miRNAs, miR5221 targeted the AUX1 gene and negatively regulate its expression in both 
sugarcane genotypes. In Fig. 7, AUX1 (CA105497) was significantly upregulated (3.27-) in YA05-179 and downregulated (0.37-) in ROC22. Constitutive triple-response 1 (CTR1) is a gene involved in the ET signal transduction pathway. A previous study showed that the amino terminus of CTR1 could combine with the ET receptor to form a complex, which negatively regulates the ET response [91]. Our analysis indicated that the expression of miR6478 was obviously repressed (0.20-) in YA05-179 but remained stable (1.14-) in ROC22. The CTR1 gene (Sugarcane_Unigene_BMK.73145), one of the predicted target genes of miR6478, was significantly upregulated (1.43-) in YA05-179 and only slightly downregulated (0.82-) in ROC22. This result indicated that miR6478 might negatively regulate CTR1 expression and affected the ET signal transduction pathway. ABA pathway has been reported to be a negative regulator of plant disease resistance [92]. $A B F$ is a binding factor of ABA-responsive element-binding protein (AREB) [93]. AREB/ABF transcription factors are ABA-responsive element-binding proteins that regulating the expression of ABA-related genes [93]. In the present study, miR5783 targeted the $A B F$ gene (Sugarcane_Unigene_BMK.68798) and slightly downregulated (0.81- and 0.88-) it in both YA05-179 and ROC22 however the level was not significant. This finding suggested that miR5783 might play a negative regulation role in $A B F$ in YA05-179, but a positive regulation role in $A B F$ in ROC22. Based on the above findings, we speculated that the upregulated and downregulated expression of differentially expressed miRNAs reflect the close relationship of the auxin signal transduction pathway, ABA signal transduction pathway, and ET signal transduction pathway in sugarcane during $S$. scitamineum infection.

\section{Pathway of plant-pathogen interaction}

In plant-pathogen interaction, plants have a variety of defense mechanisms against different pathogens [94], including hypersensitive responses (HR), changes in enzymatic activities, and the accumulation of defense proteins [95]. A previous study has indicated that catalase plays an important role in plant defense response, stress response, and in regulating redox balance in cells [96]. In the present study, miR5054 was downregulated (0.64-) in YA05-179 and significantly upregulated (3.83-) in ROC22. The expression of one miR5054 targeted gene, peroxisome 5 (PEX5, CA223872), was significantly increased (1.51-) in YA05-179 and nearly remained stable (1.12-) in ROC22. Calcium-dependent and calmodulinindependent protein kinase $(C D P K)$ is a key protein gene that involved in the mechanism of disease resistance in plants [97]. Overexpression of CDPK triggers the primary reaction of active cell necrosis. In addition, hydrogen peroxide also activates CDPK and enhances the expression of the $C D P K$ gene [98]. In the present study, $C D P K$ gene
(Sugarcane_Unigene_BMK.34960), one of the predicted target genes of miR5054, was significantly upregulated (1.67-) in YA05-179 and downregulated (0.34-) in ROC22, revealing an opposite expression trend compared to that of miR5054 in both sugarcane genotypes. These results revealed that there might be a negatively regulated mode between miR5054 and PEX5 or CDPK gene. The formation of HR depends on the interaction between plant disease resistance gene products and the avirulence (Avr) gene product of the corresponding pathogen [99]. RPM1 (effector-triggered immune receptor) is a type of R-genemediated plant disease resistance, whose overexpression induces HR in plants [99]. In the present study, miR5671 was determined to target RPM1. We found that the expression of RPM1 (Sugarcane_Unigene_BMK.42342) was significantly increased (1.53- and 2.54-) in YA05-179 and ROC22 after S. scitamineum challenge. Meanwhile, miR5671 was significantly downregulated (0.65- and 0.65) in both sugarcane genotypes, suggesting the negative regulation of $R P M 1$ in sugarcane smut resistance.

\section{Conclusions}

This is the first study that has employed highthroughput sequencing technology to identify and establish the expression profiles of various sugarcane miRNAs that are associated with $S$. scitamineum challenge. The post-transcriptional miRNA regulatory mechanism in the compatible and incompatible interactions between sugarcane and S. scitamineum was then systemically evaluated, which enriched and deepened our knowledge in the molecular mechanism underlying sugarcane resistance to smut disease. The present study has presented various regulatory pathways that are affected by smut infection, as well as generated a regulatory network of some miRNAs in sugarcane post $S$. scitamineum infection (Fig. 7).

\section{Additional files}

Additional file 1: Table S1. The forward primers of qRT-PCR performed to validate the 20 selected differentially expressed miRNAs. (DOCX $20 \mathrm{~kb}$ )

Additional file 2: Table S2. The primers of qRT-PCR performed to validate 23 selected miRNA target genes. (DOC $41 \mathrm{~kb}$ )

Additional file 3: Table S3. The filtering results of high-through sequencing data in the four libraries. (DOC $40 \mathrm{~kb}$ )

Additional file 4:Figure S1. Length distribution of the unique SRNA sequences in the four libraries. RCK and YACK: ROC22 and YA05-179 under sterile water stress after $48 \mathrm{~h}$, respectively; RT and YAT: ROC22 and YA05-179 under Sporisorium scitamineum stress after $48 \mathrm{~h}$, respectively. (TIF $35 \mathrm{~kb}$ )

Additional file 5: Table S4. The statistics of types and total number of repetitive sequence of the sRNAs in the four libraries. (DOC 69 kb)

Additional file 6: Table S5. The matching results of sRNAs among non-coding RNAs in the four libraries by Genbank search. (DOC 30 kb)

Additional file 7: Table S6. The matching results of sRNAs among non-coding RNAs in the four libraries by Rfam search. (DOC $31 \mathrm{~kb}$ ) 
Additional file 8: Figure S2. The distribution of first nucleotide bias (A) and the nucleotide bias at each position (B) of the novel miRNAs in the four libraries. (A) Each color in the figure showed the miRNA tags whose first base was a certain base. Height of bar was proportional to the frequency of the corresponding base at the given length from 20 to $23 \mathrm{nt}$. (B) Each color in the figure showed the miRNA tags whose certain base was a certain base. Height of bar was proportional to the frequency of the corresponding base at the given position from 1 to $23 \mathrm{nt}$. RCK and YACK: ROC22 and YA05-179 under sterile water stress after $48 \mathrm{~h}$, respectively; RT and YAT: ROC22 and YA05-179 under Sporisorium scitamineum stress after $48 \mathrm{~h}$, respectively. (ZIP $279 \mathrm{~kb}$ )

Additional file 9: Table S7. The significantly differentially expressed known miRNAs in the RT/RCK. (DOC $70 \mathrm{~kb}$ )

Additional file 10: Table S8. The significantly differentially expressed known miRNAs in the YAT/YACK. (DOC $37 \mathrm{~kb}$ )

Additional file 11:Table S9. The significantly differentially expressed novel miRNAs in the RT/RCK. (DOC $42 \mathrm{~kb}$ )

Additional file 12: Table S10. The significantly differentially expressed novel miRNAs in the YAT/YACK. (DOC $32 \mathrm{~kb}$ )

Additional file 13: Table S11. Prediction of target genes of known and novel miRNAs. (DOC $30 \mathrm{~kb}$ )

Additional file 14: Table S12. The prediction results for partial target genes of differentially expressed miRNAs. (DOC $41 \mathrm{~kb}$ )

Additional file 15: Figure S3. $G O$ categories and distribution of known miRNAs targets in RT/RCK (A) and YAT/YACK (B), respectively. RCK and YACK: ROC22 and YA05-179 under sterile water stress after $48 \mathrm{~h}$, respectively: RT and YAT: ROC22 and YA05-179 under Sporisorium scitamineum stress after $48 \mathrm{~h}$, respectively. (TIF $3774 \mathrm{~kb}$ )

Additional file 16: Figure S4. GO categories and distribution of novel miRNAs targets in RT/RCK (A) and YAT/YACK (B), respectively. RCK and YACK: ROC22 and YA05-179 under sterile water stress after $48 \mathrm{~h}$, respectively; RT and YAT: ROC22 and YA05-179 under Sporisorium scitamineum stress after $48 \mathrm{~h}$, respectively. (TIF $1606 \mathrm{~kb}$ )

Additional file 17: Table S13. KEGG analysis of predicted target genes of known miRNAs in RT/RCK. (DOC $58 \mathrm{~kb}$ )

Additional file 18: Table S14. KEGG analysis of predicted target genes of known miRNAs in YAT/YACK. (DOC $34 \mathrm{~kb}$ )

Additional file 19: Table S15. KEGG analysis of predicted target genes of novel miRNAs in RT/RCK. (DOC $57 \mathrm{~kb}$ )

Additional file 20: Table S16. KEGG analysis of predicted target genes of novel miRNAs in YAT/YACK. (DOC $58 \mathrm{~kb}$ )

\section{Abbreviations}

2DE: Two-dimensional gel electrophoresis; ABA: Abscisic acid; AGO: Argonaut; ANOVA: Analysis of Variance; APS1: ATP sulfurylase 1; AREB: ABA-responsive element-binding protein; AUX1: Auxin transporter; Avr: Avirulence; BLASTn: Basic local alignment search tool; BR: Brassinosteroid; CBF: C-repeat binding factor; CDNA-AFLP: CDNA-amplified fragment length polymorphism; CDPK: Calmodulin-independent protein kinase; CK: Cytokinin; CTR1: Constitutive triple-response 1; ESTs: Expressed sequence tags; ET: Ethylene; GA: Gibberellin acid; GAPDH: Glyceraldehyde-3-phosphate dehydrogenase; GO: Gene Ontology; Hc-Pro: Helper component-proteinase; HR: Hypersensitive responses; HSP72: Heat shock protein 72; IAA: Indole-3-acetic acid; iTRAQ: Isobaric tags for relative and absolute quantitation; KEGG: Kyoto Encyclopedia of Genes and Genomes; LAMP: Loop-mediated isothermal amplification; MALDI-TOF MS: Matrix-assisted laser desorption/ionization time-of-flight mass spectrometry; MAPK: Mitogen-activated protein kinase; miRNA: microRNA; PEX5: Peroxisome 5; piRNA: Piwi-interacting RNA; PKA: Kinase A; qRT-PCR: Quantitative real-time PCR; RPM: Reads per million; RPM1: Effector-triggered immune receptor; rRNA: Ribosomal RNA; SA: Salicylic acid; scRNA: Small cytoplasmic RNA; siRNAs: Small interfering RNAs; snoRNA: Small nucleolar RNA; snRNA: Small nuclear RNA; SOLiD: Sequencing by oligonucleotide ligation and detection; SRA: Sequence read archive; sRNA: Non-coding small RNA; srpRNA: Signal recognition particle RNA; SSH: Suppression subtractive hybridization; tRNA: Transfer RNA

\section{Acknowledgements}

We gratefully acknowledge Mingjie Li (Fujian Agriculture and Forestry University, Fuzhou, China) and the Beijing Genomics Institute (BGl, Shenzhen, China) for useful discussions on sRNA sequencing data analysis.

\section{Funding}

This work was supported by Natural Science Foundation of Fujian province, China (2015 J06006), the earmarked fund for the Modern Agriculture Technology of China (CARS-20), the National Natural Science Foundation of China (31501363), the Program for New Century Excellent Talents in Fujian Province University (JA14095) and the Research Funds for Distinguished Young Scientists in Fujian Agriculture and Forestry University (xjq201630). The funding bodies had no role in the design of the study and collection, analysis, and interpretation of data and in writing the manuscript.

\section{Availability of data and materials}

The data supporting the conclusions of this article are within the paper and its additional files. All sequencing reads are deposited in the National Center for Biotechnology Information under the BioProject number PRJNA322114 with the Sequence Read Archive (SRA) study accession SRP075407.

\section{Authors' contributions}

YCS, YYZ and YXQ conceived, designed and initiated the project. YYZ prepared materials. YYZ, NH, FL, WHS, WA, QBW and JLG performed experiments and contributed to data analysis and validation. YCS and YXQ drafted the manuscript. LPX and YXQ helped to revise the manuscript. All authors read and approved the final manuscript.

\section{Competing interests}

The authors declare that they have no competing interests.

\section{Consent for publication}

Not applicable.

\section{Ethics approval and consent to participate}

Not applicable. This is to confirm that no specific permits were needed for the described experiments, and this study did not involve any endangered or protected species.

\section{Publisher's Note}

Springer Nature remains neutral with regard to jurisdictional claims in published maps and institutional affiliations.

Received: 15 April 2016 Accepted: 21 April 2017

Published online: 24 April 2017

\section{References}

1. Sundar AR, Barnabas EL, Malathi P, Viswanathan R. A mini-review on smut disease of sugarcane caused by Sporisorium scitamineum. In: Mworia J, editor. Botany. Croatia: InTech Press; 2012. p. 109-28.

2. $\mathrm{Xu} \mathrm{LP}$, Chen RK. Current status and prospects of smut and smut resistance breeding in sugarcane. Fujian J Agr Sci (in Chinese). 2000;15:26-31.

3. Hsieh WH, Lee CS. Compatability and pathogenicity of two races of Ustilago scitaminea Syd. in Taiwan. Taiwan Sugar. 1978;25:46-8.

4. Heinze BS, Thokoane LN, Williams NJ. The smut sugarcane interaction as a model system for the integration of marker discovery and gene isolation programmes. P S Afr Sugar Technol Assoc. 2001;75:88-93.

5. Borrás-Hidalgo O, Thomma BPHJ, Carmona E, Borroto CJ, Pujol M, Arencibia A, Lopez J. Identification of sugarcane genes induced in disease-resistant somaclones upon inoculation with Ustilago scitaminea or Bipolaris sacchari. Plant Physiol Biochem. 2005;43:1115-21.

6. Que YX, Xu LP, Lin JW, Ruan MH, Zhang MQ, Chen RK. Differential protein expression in sugarcane during sugarcane-Sporisorium scitamineum interaction revealed by 2-DE and MALDI-TOF-TOF/MS. Com Funct Genom 2011;2011:989016

7. Su YC, Xu LP, Wang ZQ, Peng Q, Yang YT, Chen Y, et al. Comparative proteomics reveals that central metabolism changes are associated with resistance against Sporisorium scitamineum in sugarcane. BMC Genomics. 2016;17:800

8. Su YC, Yang YT, Peng Q, Zhou DG, Chen Y, Wang ZQ, et al. Development and application of a rapid and visual loop-mediated isothermal 
amplification for the detection of Sporisorium scitamineum in sugarcane. Sci Rep. 2016;6:23994.

9. Su YC, Xu LP, Xue BT, Wu QB, Guo JL, Wu LG, et al. Molecular cloning and characterization of two pathogenesis-related $\beta$-1,3-glucanase genes ScGluA1 and ScGluD1 from sugarcane infected by Sporisorium scitamineum. Plant Cell Rep. 2013;32:1503-19.

10. Su YC, Wang ZQ, Liu F, Li Z, Peng Q, Guo JL, et al. Isolation and characterization of ScGluD2, a new sugarcane beta-1,3-glucanase D family gene induced by Sporisorium scitamineum, $\mathrm{ABA}, \mathrm{H}_{2} \mathrm{O}_{2}, \mathrm{NaCl}$, and $\mathrm{CdCl}_{2}$ stresses. Front Plant $\mathrm{Sci}$. 2016;7:e0153929.

11. Su YC, Guo JL, Ling $H$, Chen SS, Wang SS, Xu LP, et al. Isolation of a novel peroxisomal catalase gene from sugarcane, which is responsive to biotic and abiotic stresses. PLoS ONE. 2014;9:e84426.

12. Su YC, Xu LP, Fu ZW, Yang YT, Guo JL, Wang SS, et al. ScChi, encoding an acidic class III chitinase of sugarcane, confers positive responses to biotic and abiotic stresses in sugarcane. Int J Mol Sci. 2014;15:2738-60.

13. Wu QB, Xu LP, Guo JL, Su YC, Que YX. Transcriptome profile analysis of sugarcane responses to Sporisorium scitaminea infection using solexa sequencing technology. BioMed Res Int. 2013;2013:298920.

14. Que YX, Xu LP, Wu QB, Liu YF, Ling H, Liu YH, et al. Genome sequencing of Sporisorium scitamineum provides insights into the pathogenic mechanisms of sugarcane smut. BMC Genomics. 2014;15:996.

15. Taniguti LM, Schaker PD, Benevenuto J, Peters LP, Carvalho G, Palhares A, et al. Complete genome sequence of Sporisorium scitamineum and biotrophic interaction transcriptome with sugarcane. PLOS ONE. 2015;10:e0129318.

16. Dutheil JY, Mannhaupt G, Schweizer G, Sieber CM, Münsterkötter M, Güldener $U$, et al. A tale of genome compartmentalization: the evolution of virulence clusters in smut fungi. Genome Biol Evol. 2016:8:681-704.

17. Kämper J, Kahmann R, Bölker M, Ma LJ, Brefort T, Saville BJ, et al. Insights from the genome of the biotrophic fungal plant pathogen Ustilago maydis. Nature. 2006:444:97-101.

18. Laurie JD, Ali S, Linning R, Mannhaupt G, Wong P, Güldener U, et al. Genome comparison of barley and maize smut fungi reveals targeted loss of RNA silencing components and species-specific presence of transposable elements. Plant Cell. 2012;24:1733-45.

19. Schirawski J, Mannhaupt G, Münch K, Brefort T, Schipper K, Doehlemann G, et al. Pathogenicity determinants in smut fungi revealed by genome comparison. Science. 2010;330:1546-8.

20. Yang $L$, Huang $H$. Roles of small RNAs in plant disease resistance. J Integr Plant Boil. 2014:56:962-70.

21. Jones MR, Quinton LJ, Blahna MT, Neilson JR, Fu S, Ivanov AR, et al. Zcchc11-dependent uridylation of microRNA directs cytokine expression. Nat Cell Biol. 2009:11:1157-63.

22. Kim J, Inoue K, Ishii J, Vanti WB, Voronov SV, Murchison E, et al. A microRNA feedback circuit in mid brain dopa mine neurons. Science. 2007:317:1220-4.

23. Lee $\mathrm{RC}$, Feinbaum RL, Ambros $V$. The C.elegans heterochronic gene lin-4 encodes small RNAs with antisense complementarity to lin-14. Cell. 1993;75:843-54

24. Reinhart BJ, Slack FJ, Basson M. The 21-nucleotide le-t 7 RNA regulates developmental timing in Caenorhabditis elegans. Nature. 2000;403:901-6.

25. Khraiwesh B, Arif MA, Seumel Gl, Ossowski S, Weigel D, Reski R, et al. Transcriptional control of gene expression by microRNAs. Cell. 2010;140:111-22

26. Giraldez AJ, Cinalli RM, Glasner ME, Enright AJ, Thomson JM, Baskerville S, et al. MicroRNAs regulate brain morphogenesis in zebra fish. Science. 2005; 308:833-8

27. Krützfeldt J, Rajewsky N, Braich R, Rajeev KG, Tuschl T, Manoharan M, et al. Silencing of microRNAs in vivo with Antagomirs. Nature. 2005;438:685-9.

28. Ashraf SI, McLoon AL, Sclarsic SM, Kunes S. Synaptic protein synthesis associated with memory is regulated by the risc pathway in Drosophila. Cell. 2006;124:191-205.

29. Johnston RJ, Hobert O. A microRNA controlling left/right neurona asymmetry in Caenorhabditis elegans. Nature. 2003:426:845-9.

30. Zamore PD, Haley B. Ribo-gnome: The big world of small RNAs. Science. 2005;309:1519-24.

31. Guo HS, Xie Q, Fei JF. MicroRNA directs mRNA cleavage of the transcription factor $\mathrm{NaCl}$ to downregulate auxin signals for Arabidopsis lateral root development. Plant Cell. 2005;17:1376-86.

32. Wang JW, Wang L, Mao YB. Control of root cap formation by microRNAtargeted auxin response factors in Arabidopsis. Plant Cell. 2005;17:2204-16.
33. Bai $S$, Saito $T$, Ito A, Tuan PA, Xu Y, Teng Y, et al. Small RNA and PARE sequencing in flower bud reveal the involvement of sRNAs in endodormancy release of Japanese pear (Pyrus pyrifolia 'Kosui'). BMC Genomics. 2016;17:230.

34. Llave C, Kasschau KD, RectorM A. Endogenous and silencing-associated small RNAs in plants. Plant Cell. 2002;14:1605-19.

35. Napoli C, Lemieux C, Jorgensen R. Introduction of a chimeric chalcone synthase gene into petunia results in reversible co-suppression of homologous genes in trans. Plant Cell. 1990;2:279-89.

36. Sunkar R, Zhu JK. Novel and stress-regulated microRNAs and other small RNAs from Arabidopsis. Plant Cell. 2004;16:2001-19.

37. Zhou LG, Liu YH, Liu ZC, Gong DY, Luo LY. Genome-wide identification and analysis of drought-responsive microRNAs in oryza sativa. J Exp Bot. 2010;61:4157-68.

38. Jones-Rhoades MW, Bartel DP. Computational identification of plant microRNAs and their targets, including a stress-induced miRNA. Mol Cell. 2004;14:787-99.

39. Patade $\mathrm{V}$, Suprasaima P. Short-term salt and PEG stresses regulate expression of microRNA miR159 in sugarcane leaves. J Crop Sci Biotechnol. 2010;13:177-82

40. Lu S, Sun Y, Shi R, Clark C, Li L, Chiang VL. Novel and mechanical stressresponsive microRNAs in Populus trichocarpa that are absent from Arabidopsis. Plant Cell. 2005;17:2186-203.

41. Chapman EJ, Prokhnevsky Al, Gopinath K, Dolja W, Carrington JC. Viral RNA silencing suppressors inhibit the microRNA pathway at an intermediate step. Gene Dev. 2004;18:1179-86.

42. Kasschau KD, Xie Z, Allen E, Llave C, Chapman EJ, Krizan KA, et al. P1/HCPro, a viral suppressor of RNA silencing, interferes with Arabidopsis development and miRNA function. Dev Cell. 2003:4:205-17.

43. Jin SB, Fu HT, Jiang SF, Xiong YW, Qiao H, Zhang WY, et al. Identification of androgenic gland microRNA and their target genes to discover sex-related microRNA in the oriental river prawn, Macrobrachium nipponense. Genet Mol Res. 2015;14:18396-406.

44. Hong XS, Qin JH, Chen R, Yuan LL, Zha JM, Wang ZJ. Identification and characterization of novel and conserved microRNAs in several tissues of the Chinese rare minnow (Gobiocypris rarus) based on illumina deep sequencing technology. BMC Genomics. 2016;17:1-11.

45. Bukhari SAH, Shang SH, Zhang M, Zheng WT, Zhang GP, Wang TZ, et al. Genome-wide identification of chromium stress-responsive micro RNAs and their target genes in tobacco (Nicotiana tabacum) roots. Environ Toxicol Chem. 2015;34:2573-82

46. Calabrese JM, Seila AC, Yeo GW, Sharp PA. RNA sequence analysis defines dicer's role in mouse embryonic stem cells. Proc Natl Acad Sci. 2007;104: 18097-102.

47. Que YX, Su YC, Guo JL, Wu QB, Xu LP. A global view of transcriptome dynamics during Sporisorium scitamineum challenge in sugarcane by RNAseq. PLOS ONE. 2014:9:e106476.

48. Yang RR, Zeng YL, Yi XY, Zhao LJ, Zhang YF. Small RNA deep sequencing reveals the important role of microRNAs in the halophyte Halostachys caspica. Plant Biotechnol J. 2015;13:395-408.

49. Xie FL, Jones DC, Wang QL, Sun RR, Zhang BH. Small RNA sequencing identifies miRNA roles in ovule and fibre development. Plant Biotechnol J. 2015:13:355-69.

50. Long RC, Li MN, Kang JM, Zhang TJ, Sun Y, Yang QC. Small RNA deep sequencing identifies novel and salt-stress-regulated microRNAs from roots of Medicago sativa and Medicago truncatula. Physiol Plantarum. 2015;154:13-27.

51. Audic S, Claverie JM. The significance of digital gene expression profiles. Genome Res. 1997;7:986-95.

52. Zhang YX, Zhou X, Ge X, Jiang JH, Li F, Huang YP. Insect-specific microRNA involved in the development of the silkworm Bombyx mori. PLOS ONE. 2009:4:e4677.

53. Allen E, Xie Z, Gustafson AM, Carrington JC. MicroRNA-directed phasing during trans-acting siRNA biogenesis in plants. Cell. 2005;121:207-21.

54. Schwab R, Javier FP, Markus R, Carla S, Markus S, Detlef W. Specific effects of microRNAs on the plant transcriptome. Dev Cell. 2005:8:517-27.

55. Xu LN, Ling YH, Wang YQ, Wang ZY, Hu BJ, Zhou ZY, et al. Identification of differentially expressed microRNAs between Bacillus thuringiensis Cry1Abresistant and -susceptible strains of Ostrinia furnacalis. Sci Rep. 2015;5:15461. 
56. Jiang Z. Identification and functional analysis of miRNAs and important target genes from process of maize callus induction, Dr. Phil. thesis, Sichuan Agricultural University. 2013.

57. Livak KJ, Schmittgen TD. Analysis of relative gene expression data using real-time quantitative $P C R$ and the $2^{-\Delta \Delta C T}$ method. Methods. 2001:25:402-8.

58. Que YX, Xu LP, Xu JS, Zhang JS, Zhang MQ, Chen RK. Selection of control genes in real-time $q P C R$ analysis of gene expression in sugarcane. Chinese J Trop Crop. 2009;30:274-8.

59. Hafner MP, Landgraf P, Ludwig J, Rice A, Ojo T, Lin C. Identification of microRNAs and other small regulatory RNAs using cDNA library sequencing. Methods. 2008;44:3-12.

60. Ahmed F, Senthil-Kumar M, Lee S, Dai X, Mysore KS, Zhao PX. Comprehensive analysis of small RNA-seq data reveals that combination of miRNA with its isomiRs increase the accuracy of target prediction in Arabidopsis thaliana. RNA Biol. 2014;11:1414-29.

61. Flávia T, Clícia G, Mariana CB, Cristian AR, Paulo CF. Computational identification and analysis of novel sugarcane microRNA. BMC Genomics. 2012;13:290.

62. Dalvi SG, Vasekar VC, Yadav A, Tawar PN, Dixit GB, Prasad DT, et al. Screening of promising sugarcane somaclones for agronomic traits, and smut resistance using PCR amplification of inter transcribed region (ITS) of Sporisorium scitaminae. Sugar Tech. 2012;14:68-75.

63. Li J, Wang Z, Yang BP. Identification of smut resistance in transgenic sugarcane. Guangxi Agric Sci. 2009;40:1150-5.

64. Metzker ML. Sequencing technologies the next generation. Nat Rev Genet 2010;11:31-46.

65. Xia R, Zhu H, An YQ, Beers EP, Liu ZR. Apple miRNAs and tasiRNAs with novel regulatory networks. Genome Biol. 2012;13:R47.

66. Zhang XN, Li X, Liu JH. Identification of conserved and novel cold-responsive microRNAs in trifoliate orange (Poncirus trifoliata L.) using high-throughput sequencing. Plant Mol Biol Rep. 2014;32:328-41.

67. Sun LM, Ai XY, Li WY, Guo WW, Deng XX. Identification and comparative profiling of miRNAs in an early flowering mutant of trifoliate orange and its wild type by genome-wide deep sequencing. PLoS ONE. 2012;7:e43760

68. Wei R, Qiu D, Wilson IW, Zhao H, Lu S, Miao J, et al. Identification of novel and conserved microRNAs in Panax notoginseng roots by high-throughput sequencing. BMC Genomics. 2015;16:835.

69. Manners JM. Functional genomics of sugarcane. Adv Bot Res. 2011;60:91-168

70. Palatnik JF, Allen E, Wu X. Control of leaf morphogenesis by microRNAs. Nature. 2003;425:257-63.

71. Winter J, Jung S, Keller S, Gregory RI, Diederichs S. Many roads to maturity: microRNA biogenesis pathways and their regulation. Nat Cell Biol. 2009;11: 228-34.

72. Zhu J, Yang CJ, Wang J. Real-time fluorescent quantitative PCR and application in scientific research. Biotechnol Bull. 2009;2:73-6.

73. Luo YJ. Identification and characterization of microRNAs from Chinese PCNA persimmon based on high throughput sequencing, Master's degree dissertation, Huazhong Agricultural University. 2014.

74. Tang MM, Chen H, Zhang J, Ren JY, Xu N. Application of next generation sequencing in microRNA detection. Hereditas. 2012;34:784-92.

75. Wu L, Zhang QQ, Zhou HY, Ni FR, Wu XY, Qi YJ, et al. Rice microRNA effector complexes and targets. Plant Cell. 2009:21:3421-35.

76. Zhang ZM, Song R, Peng H. Bioinformatic prediction of microRNAs and their target genes in maize. Acta Agron Sin. 2010;36:1324-35.

77. Coram TE, Settles ML, Chen X. Transcriptome analysis of hightemperature adult-plant resistance conditioned by Yr39 during the wheat-Puccmia striiformis f. sp. tritici interaction. Mol Plant Pathol. 2008:9:479-93.

78. Bolton MD, Kolmer JA, Xu WW. Lr34-mediated leaf rust resistance in wheat: transcript profiling reveals a high energetic demand supported by transient recruitment of multiple metabolic pathways. Mol Plant Microbe In. 2008;21:1515-27.

79. Wong J, Gao L, Yang Y, Zhai JX, Arikit S, Yu Y, et al. Roles of small RNAs in soybean defense against Phytophthora sojae infection. Plant J. 2014;79:928-40

80. $\mathrm{Li} \mathrm{CW}$. The cytological and transcriptomic investigations into interactions of tomato and powdery mildew, Dr. Phil. thesis, Chiese Academy of Agricultural Science. 2007.

81. Ichimura K. Mogen-activated protein kinase cascades in plans: a new nomenclature. Trends Plant Sci. 2002;7:301-8.
82. Fu SF, Chou WC, Huang DD. Transcriptional regulation of a rice mitogenactivated protein kinase gene OsMAPK4 in response to environmental stress. Plant Cell Physiol. 2002;43:958-63.

83. Angelo G, Lee H, Weiner RI. CAMP-dependent protein kinase inhibits the mitogenic action of vascular endothelial growth factor and fibroblast growth factor in capillary endothelial cells by blocking Raf activation. J Cell Biochem. 1997;67:353-66.

84. Reynolds LP, Allen GV. A review of heat shock protein induction following cerebellar injury. Cerebellum. 2003;2:171-7.

85. Zhang BH, Pan XP, Wang QL, Cobb GP, Anderson TA. Identification and characterization of new plant microRNAs using EST analysis. Cell Res. 2005; 15:336-60.

86. Bari $\mathrm{R}$, Jones JD. Role of plant hormones in plant defence responses. Plant Mol Biol. 2009;69:473-88.

87. Zhou JX, Hu XW, Zhang HW. Regulatory role of ABA in plant response to biotic stresses. J Agr Biotechnol. 2008;16(1):169-74

88. Wang SK, Bai YH. Auxin-related gene families in abiotic stress response in Sorghum bicolor. Func Integr Genomic. 2010;10:533-46.

89. Swarup R, Friml J, Marchant A, Ljung K, Sandberg G, Palme K, et al. Localization of the auxin permease AUX1 suggests two functionally distinct hormone transport pathways operate in the Arabidopsis root Apex. Gene Dev. 2001;15:2648-53.

90. Park JE, Park JY, Kim YS. GH3-mediated auxin homeostasis links growth regulation with stress adaptation response in Arabidopsis. J Biol Chem. 2007;13:10036-46

91. Gao Z, Chen YF, Randlett MD. Localization of the Raf-like kinase CTRI to the endoplasmic reticulum of Arabidopsis through participation in ethylene receptor signaling complexes. J Biol Chem. 2003;278:34725-32.

92. Fan J, Hill L, Crooks C, Doerner P, Lamb C. Abscisic acid has a key role in modulating diverse plant-pathogen interactions. Plant Physiol. 2009;150:1750-61.

93. Hong L, Liu X, Li L. AREB/ABF transcription factors and their involvement in ABA signal transduction. Plant Physiol J. 2011;47:211-7.

94. Boller T. Induction of hydrolases as defense reaction against pathogens. In: Key JL, Kosuge T, editors. Mol Cell Biol. New York: AR Liss; 1985. p. 247-62.

95. Takken FLW, Joosten MHAJ. Plant resistance genes: their structure, function and evolution. Eur J Plant Pathol. 2000;106:699-713.

96. Mittler R, Herr EH, Orvar BL, Van Camp W, Willekens H, Inzé D, et al. Transgenic tobacco plants with reduced capability to detoxify reactive oxygen intermediates are hyperresponsive to pathogen infection. Proc Natl Acad Sci. 1999:96:14165-70.

97. Lee J, Rudd JJ. Calcium-dependent protein kinases: versatile plant signalling components necessary for pathogen defence. Trends Plant Sci. 2002;7:97-8.

98. Chico JM, Raices M, Tellez-inon MT. A calcium-dependent protein kinase is systemically induced upon wounding in tomato plants. Plant Physiol. 2002;128:256-70.

99. Keller H, Pamboukdjian N, Pouchet M. Pathogen-induced elicitin production in transgenic tobacco generates a hypersensitive response and nonspecific disease resistance. Plant Cell. 1999;11:223-36.

\section{Submit your next manuscript to BioMed Central and we will help you at every step:}

- We accept pre-submission inquiries

- Our selector tool helps you to find the most relevant journal

- We provide round the clock customer support

- Convenient online submission

- Thorough peer review

- Inclusion in PubMed and all major indexing services

- Maximum visibility for your research

Submit your manuscript at www.biomedcentral.com/submit 